\title{
Evidence for different accretion regimes in GRO J1008-57
}

\author{
Matthias Kühnel $^{1}$, Felix Fürst ${ }^{2,3}$, Katja Pottschmidt ${ }^{4,5}$, Ingo Kreykenbohm ${ }^{1}$, Ralf Ballhausen ${ }^{1}$, Sebastian Falkner ${ }^{1}$, \\ Richard E. Rothschild ${ }^{6}$, Dmitry Klochkov ${ }^{7}$, and Jörn Wilms ${ }^{1}$
}

\author{
1 Dr. Karl Remeis-Observatory \& ECAP, Universität Erlangen-Nürnberg, Sternwartstr. 7, 96049 Bamberg, Germany \\ e-mail: matthias . kuehnel@sternwarte.uni-erlangen. de \\ 2 Cahill Center for Astronomy and Astrophysics, California Institute of Technology, Pasadena, CA 91125, USA \\ 3 European Space Astronomy Centre (ESA/ESAC), Operations Department, Villanueva de la Cañada, 28692 Madrid, Spain \\ ${ }^{4}$ CRESST/CSST/Department of Physics, UMBC, Baltimore, MD 21250, USA \\ 5 NASA Goddard Space Flight Center, Greenbelt, MD 20771, USA \\ ${ }^{6}$ Center for Astrophysics and Space Sciences, University of California, San Diego, La Jolla, CA 92093, USA \\ 7 Institut für Astronomie und Astrophysik, Universität Tübingen, Sand 1, 72076 Tübingen, Germany
}

Received 16 June 2016 / Accepted 14 August 2017

\begin{abstract}
We present a comprehensive spectral analysis of the BeXRB GRO J1008-57 over a luminosity range of three orders of magnitude using NuSTAR, Suzaku, and RXTE data. We find significant evolution of the spectral parameters with luminosity. In particular, the photon index hardens with increasing luminosity at intermediate luminosities in the range $10^{36}-10^{37} \mathrm{erg} \mathrm{s}^{-1}$. This evolution is stable and repeatedly observed over different outbursts. However, at the extreme ends of the observed luminosity range, we find that the correlation breaks down, with a significance level of at least $3.7 \sigma$. We conclude that these changes indicate transitions to different accretion regimes, which are characterized by different deceleration processes, such as Coulomb or radiation breaking. We compare our observed luminosity levels of these transitions to theoretical predications and discuss the variation of those theoretical luminosity values with fundamental neutron star parameters. Finally, we present detailed spectroscopy of the unique "triple peaked" outburst in 2014/15 which does not fit in the general parameter evolution with luminosity. The pulse profile on the other hand is consistent with what is expected at this luminosity level, arguing against a change in accretion geometry. In summary, GRO J1008-57 is an ideal target to study different accretion regimes due to the well-constrained evolution of its broad-band spectral continuum over several orders of magnitude in luminosity.
\end{abstract}

Key words. X-rays: binaries - stars: neutron - stars: emission-line, Be - pulsars: individual: GRO J1008-57

\section{Introduction}

The strong magnetic field of an accreting and pulsing neutron star channels accreted matter onto its magnetic poles. There, infalling plasma with velocities close to the speed of light is decelerated to rest on the surface. The X-ray spectrum of accreting neutron stars is therefore dominated by strong bulk-motion Comptonizaton caused by the interaction of primary soft X-rays from the accretion mound or column with hot electrons in the accretion column. A self-consistent model of the emerging X-ray spectrum requires relativistic magnetohydrodynamical calculations of this extreme plasma and is still outside of the range of capabilities of current computing facilities. However, in recent years some progress has been made and models have become available in which the radiative transfer equations are solved assuming thermal and bulk Comptonization (Becker \& Wolff 2007; Marcu et al. 2015; Wolff et al. 2016) and which allow for different (phenomenological) velocity profiles (Farinelli et al. 2012, 2016). The details of the physical mechanisms decelerating the accreted material to rest above the neutron star's surface are the topic of current theoretical investigations (see, e.g., discussions by Staubert et al. 2007; and Becker et al. 2012).

An ideal laboratory to investigate and check different theoretical predictions about accretion physics is the class of transient Be X-ray binaries (BeXRBs). Their luminosity changes by orders of magnitude during an outburst and the spectral evolution of the neutron star in different luminosity regimes can be studied in great detail. In previous works, using all available RXTE data, we have shown that the BeXRB GRO J1008-57 shows a tight correlation of its spectral shape with the $15-50 \mathrm{keV}$ luminosity, which appears to be stable between outbursts (Kühnel et al. 2013, 2014, hereafter K13 and K14). GRO J1008-57 was discovered with the Compton Gamma Ray Observatory (CGRO) during a luminous outburst in 1993 July (Wilson et al. 1994; Stollberg et al. 1993). The system consists of a neutron star on a wide, eccentric orbit ( $a \sin i=530$ lt-s, $e=0.68$, Coe et al. 2007) around a B0e type companion star (Coe et al. 1994). In addition to the high eccentricity, the orbital period of $P_{\text {orb }}=$ $249.48 \mathrm{~d}$ (K13) is long enough for the circumstellar disk of the Be companion to be tidally truncated at the $7: 1$ or $8: 1$ resonance (Okazaki \& Negueruela 2001), leading to regular type-I outbursts. The source showed a type-II giant outburst in 2012 November at an orbital phase of $\sim 0.3$ with a peak luminosity around $1 \mathrm{Crab}$ in the $15-50 \mathrm{keV}$ range (Fig. 1). After this outburst, GRO J1008-57 showed three regular type-I outbursts, the last of these on 2014 September (Nakajima et al. 2014a). This expected outburst was followed by two unusual type-II outbursts in 2014 November and 2015 January, occurring within the same orbit (Nakajima et al. 2014b; Kretschmar et al. 2015, and Fig. 1). To our knowledge, this "triple-peaked" outburst behavior had not been seen in any other source, although some sources, such as A 0535+262 (see, e.g., Caballero et al. 2013, and references therein) or GX 304-1 (Nakajima et al. 2012; Postnov et al. 2015b), feature rare "double-peaked" outbursts. 


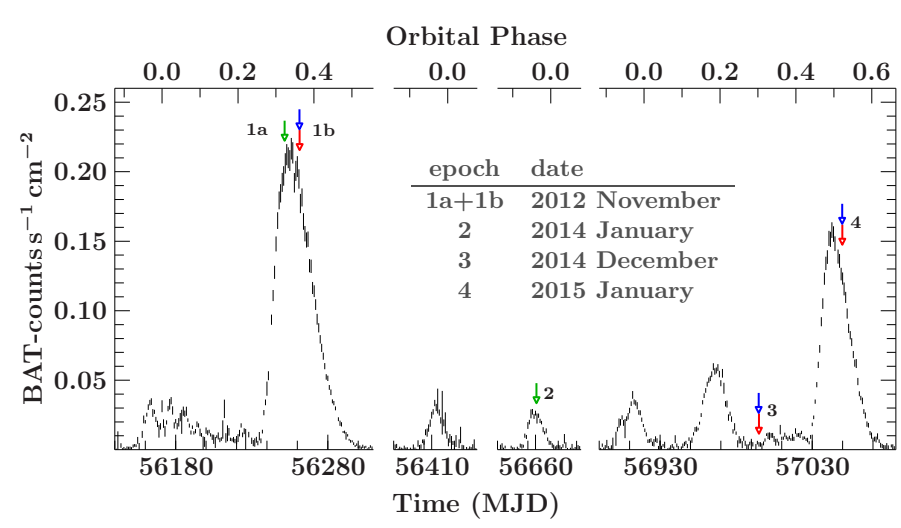

Fig. 1. Swift-BAT (Krimm et al. 2013) light curve showing the activity of GRO J1008-57 between 2012 August and 2015 February. The arrows on top mark the times of observations by Swift (red), Suzaku(green), and NuSTAR (blue). The numbers next to these arrows are the data epochs as defined in Table 1. The labeled dates correspond to these epochs.

What causes the source to undergo outbursts far away from periastron is not yet understood. A promising scenario proposed by Okazaki et al. (2013) is an inclined Be disk with respect to the orbital plane.

In this work, we compare our previous findings to observations performed by Suzaku, the Nuclear Spectroscopic Telescope Array (NuSTAR), and Swift from 2012 to 2015 in order to discuss implications on the theory of mass accretion onto magnetized neutron stars. Section 2 describes the data reduction of the observations and energy selection criteria applied to the resulting spectra. In addition, we discuss calibration uncertainties detected in the spectra. The actual spectral analysis of GRO J1008-57 is presented in Sect. 3 and the corresponding individual results and the outburst behavior of the source are discussed in Sect. 4. Section 5 focuses on the spectral evolution of GRO J1008-57, where we compare the results of the spectral analysis with our previous work and recent theoretical work. We conclude the paper with the discovery of different accretion regimes in this source.

\section{Observations and data reduction}

Figure 1 shows the light curves of GRO J1008-57 for all outbursts between 2012 August and 2016 February as recorded by the Swift Burst Alert Telescope (BAT). Arrows indicate the observations studied here (see also Table 1). During the peak of the type II giant outburst in 2012 November, GRO J1008-57 was observed with $N u S T A R, S u z a k u$, and Swift. After this type-II outburst, the source went back to its normal behavior, featuring type-I outbursts at every periastron passage. Suzaku-observed the peak of such an outburst in 2014 January. The next observation by NUSTAR, which was simultaneous with Swift, was performed in 2014 December, after the second outburst of the triplepeaked outburst. Finally, NuSTAR and Swift also performed a joint observation slightly after the peak of the third outburst, which reached around 700 mCrab in Swift-BAT during 2015 January.

The light curve of GRO J1008-57 during 2005, as measured by the Proportional Counter Array (PCA, Jahoda et al. 1996) onboard RXTE (ObsID 90089-03-02-01) was used for the pulse profile analysis presented in Sect. 4.2. The GoodXenon events of
Table 1. Observations used in this work.

\begin{tabular}{lllll}
\hline \hline Sat. $^{a}$ & ObsID & Start (MJD) & Expt. $^{b}$ & $E^{c}$ \\
\hline Suzaku & 907006010 & 56251.63 & 9061 & $1 \mathrm{a}$ \\
NuSTAR & 80001001002 & 56261.36 & 14767 & $1 \mathrm{~b}$ \\
Swift & 00031030018 & 56261.53 & 1716 & $1 \mathrm{~b}$ \\
Suzaku & 408044010 & 56660.66 & 15322 & 2 \\
NuSTAR & 90001003002 & 56994.82 & 26861 & 3 \\
Swift & 00081425001 & 56995.00 & 2263 & 3 \\
NuSTAR & 90001003004 & 57049.74 & 17260 & 4 \\
Swift & 00081425002 & 57049.79 & 1943 & 4 \\
\hline
\end{tabular}

Notes. Data from epoch 1a has been used and analyzed by Yamamoto et al. (2013, 2014) as well as by Bellm et al. (2014), who used and analyzed epoch $1 \mathrm{~b}$ in addition. ${ }^{(a)}$ Name of the X-ray mission. ${ }^{(b)}$ Exposure time in seconds. For NuSTAR the longest FPM and for Suzaku the longest XIS exposure is given. ${ }^{(c)}$ Data epoch indicating which observations have been combined in the spectral analysis.

the Proportional Counter Units (PCU) 2 and 3 were accumulated into a light curve with a time resolution of $1 \mathrm{~s}$. Finally, the light curve was transformed into the barycenter of the solar system (see K13, for more details about the extraction of RXTE light curves).

\subsection{Suzaku}

We reprocessed the Suzaku (Mitsuda et al. 2007) data following the Data Reduction (or ABC) Guide (ABC Guide, ISAS/JAXA et al. 2013). The data have been calibrated using aepipeline as distributed by HEASOFT v6.15.1. For the data of the Suzaku X-ray Imaging Spectrometers (XIS; Koyama et al. 2007), the XIS calibration database (CALDB) released on 2014-12-22 was used. For the calibration of the corresponding mirrors of the X-ray Telescopes (XRT; Serlemitsos et al. 2007), the releases 2011-05-05 (effective area) and 2009-06-05 (point spread function, PSF) are used. The High X-ray Detector (HXD; Takahashi et al. 2007) data were calibrated based on HXD CALDB release 2011-08-19.

Three of the four XIS (0,1, and 3; data from XIS2 were no longer available) were operated in the $1 / 4$ window mode during the 2012 November and 2014 January observations of GRO J1008-57. After extracting an image for each XIS and all editing modes using xselect, we applied the attitude correction by aeattcor 2 using a preliminary source region. The final source spectra of GRO J1008-57 were extracted with xselect using a circular source region centered at the source. The radii were set to $80^{\prime \prime}$ in XIS0 and XIS3 for observation 408044010 and $90^{\prime \prime}$ otherwise. Pile-up needed to be investigated for each XIS and editing mode separately and was calculated for each pixel using pileest. During observation 408044010 pile-up of more than $4 \%$ was avoided by annuli source regions with inner radii of $13-30^{\prime \prime}$. Due to the very high count rate during observation 907006010, ellipsoidal areas within the source region with more than $2 \%$ pile-up were excluded. All background regions were chosen to avoid source photons from the area covered by the Point Spread Function (PSF) of the XRT with radii of $90^{\prime \prime}$. With these regions as input for xselect, the XIS-spectra of the source and background were extracted. The redistribution matrix function (RMF) and ancillary response function (ARF) were generated with xisrmfgen and xissimarfgen, respectively. For the spectral analysis we added the spectra of the different editing modes, " $3 \times 3^{\prime \prime}$ and " $5 \times 5^{\prime \prime}$, to generate one spectrum for each XIS. 
The HXD (Kokubun et al. 2007) consisted of two detectors, the PIN diodes and the gadolinium silicate crystals (GSO). Spectra for both instruments were extracted using hxdpinxbpi and hxdgsoxbpi, respectively. These tools generate the background spectra based on the non X-ray background (NXB) using modeled events of the "tuned" background v2.2 (PIN) and v2.6 (GSO), and cosmic X-ray background (CXB), simulated following Boldt (1987). For PIN we used epoch number 11 (2011-0601) and for GSO epoch 2010-05-24 for the response file (RSP). Additionally, the GSO ARF, calibrated on the Crab pulsar from epoch 2010-05-26, was used.

We restricted the energy range of the Suzaku observations (epochs 1a and 2, see Table 1) to $0.8-10 \mathrm{keV}$ for the XISs, to $15-70 \mathrm{keV}$ for PIN, and to $60-100 \mathrm{keV}$ for GSO. The XISspectra have been rebinned following Nowak et al. (2011), that is, each energy bin has at least a minimum signal-to-noise (SNR) of 8 and a minimum number of channels close to the half-width half-maximum of the spectral resolution. For PIN and GSO, we applied a channel binning resulting in a minimum SNR of 15 and 5 in each energy bin, respectively.

During a preliminary spectral analysis we investigated possible calibration uncertainties of the Suzaku data. In both epochs, residual features in all XISs are found around the Au-edge at 2.19-2.37 keV, as already noticed by Nowak et al. (2011). Consequently, we ignored this energy range during the spectral analysis. Further calibration uncertainties around the Si-edge at $1.72-1.88 \mathrm{keV}$ are known. Using the newer calibration, however, this calibration feature is only present in the backside illuminated XIS1, which we ignored appropriately in the analysis. In epoch 2 (2014 January), the front-illuminated XIS0 and XIS3 show residual emission at energies above $9 \mathrm{keV}$ in contrast to XIS1. Thus, we ignored energies above $9 \mathrm{keV}$ in these XISs. In addition, we found a slight discrepancy of the power-law photon index, $\Gamma$, determined by the spectrum of the back-illuminated XIS1 to the other XISs in epoch 2. This behavior is already known (see, e.g., Sect. 5.2.1 of Tsujimoto et al. 2011); the photon index shift of $\Delta \Gamma=0.06_{-0.07}^{+0.04}$ is, however, only significant on the $\sim 90 \%$ confidence level.

For epoch 1a we ignored PIN data below $20 \mathrm{keV}$ because of a mismatch to NuSTAR as seen by Bellm et al. (2014), which is probably noise contamination due to the leakage current of the PIN sensors. Furthermore, we found a broad-band wave-like structure in PIN with an amplitude of $2-16 \%$ in flux, which can be described by a Gaussian absorption centered at $51.1_{-5.8}^{+2.0} \mathrm{keV}$. Kokubun et al. (2007) noticed this feature in data of the Crab pulsar (in epoch 1a, GRO J1008-57's flux was around $1 \mathrm{Crab}$ ), which they attributed to an insufficient modeling of the gadolinium fluorescent lines produced in the detector. During the spectral analysis of GRO J1008-57 we modeled this feature by the Gaussian as described above. In both epochs, the GSO-data in the four energy bins between 75 and $81 \mathrm{keV}$ scatter strongly around any predicted model flux. Because the GSO background in this energy range is dominated by the decay of activated ${ }^{151} \mathrm{Gd}$ and ${ }^{149} \mathrm{Eu}$ (Kokubun et al. 1999) we ignored those bins in our analysis.

Among the calibration features we have described above and taken into account during the spectral fitting, we added $1 \%$ systematic uncertainties to the XIS-, PIN-, and GSO-spectra to achieve a reduced $\chi^{2}$ around unity. We have followed a slightly different treatment of calibration uncertainties compared to Bellm et al. (2014). In particular, they have not taken the Gaussian absorption feature caused by the gadolinium fluorescent lines or the photon index shift in XIS1 into account, but added higher systematic uncertainties of $3 \%$ to the spectra.
However, their fit of the Suzaku data during GRO J1008-57's giant outburst (epoch 1a) still resulted in a reduced $\chi^{2}$ of 3.15 (see their Table 2).

\subsection{NUSTAR}

Extraction of the NUSTAR data was performed separately for Focal Plane Module (FPM) A and B, following the NuSTAR Data Analysis Software Guide (Perri et al. 2015). The data were extracted using the standard nustardas pipeline (v1.4.1) and CALBD 20150316 as distributed with HEASOFT v6.16 and cleaned for source occultation by the Earth and SAA passages. Spectra in mode 01 (SCIENCE) event files were extracted for each of the three observations separately using a region with $120^{\prime \prime}$ diameter, centered on the J2000 coordinates of GRO J1008-57. The background was extracted from a region of the same size at the other end of the field of view to avoid contamination from source photons in the outer wings of the PSF. We additionally extracted data from mode 06 (SCIENCE_SC), in which the optical bench star tracker is occulted, and therefore the sky image reconstruction is solely based on the spacecraft attitude. This results in a smeared point source in the sky image. However, as the metrology system is still functional, the effective area is calculated correctly (see Walton et al. 2016, for details about mode 06 extract). We used a source region with 150 " diameter to compensate for the increased apparent source size in the sky image. The mode 06 data added 16\%, 20\%, and $35 \%$ exposure time for epoch $1 \mathrm{~b}, 3$, and 4 , respectively. We carefully checked that there were no significant differences between the mode 01 and 06 data and then combined them using addascaspec.

Although the NuSTAR and Suzaku data of the type-II giant outburst of GRO J1008-57 in 2012 November have already been published by Bellm et al. (2014), we analyze these data again for two reasons. First, our previous analysis of the spectral dependence on the source's luminosity does not include these observations since the flux was higher than what had been observed until then. Secondly, by adding the mode 06 data, our statistical quality is improved compared to the spectra used by Bellm et al. (2014).

NuSTAR-FPMA and B data were fitted simultaneously between 4 and $78 \mathrm{keV}$. A first investigation of the spectra showed a significant mismatch between Swift and NuSTAR data below $4 \mathrm{keV}$ across all epochs, which is why we did not use NuSTAR data down to $3 \mathrm{keV}$. This mismatch has been reported as well by Bellm et al. (2014) for epoch 1b. The NUSTAR data were rebinned to a signal-to-noise-ratio of 18 in the range $4-45 \mathrm{keV}$ and 6 above $45 \mathrm{keV}$.

The barycentered NuSTAR-FPMA light curve of GRO J1008-57 during epoch 3 was extracted from the source region as defined above and with a $1 \mathrm{~s}$ time resolution.

\subsection{Swift}

The data of the X-Ray Telescope (XRT; Burrows et al. 2005; Godet et al. 2007) onboard Swift (Gehrels et al. 2004, 2005) were reprocessed following the Data Reduction Guide (Capalbi et al. 2005).

The observations of epoch $1 \mathrm{~b}$ and 4 were operated in "Windowed Timing" mode (WT) and that of epoch 3 in "Photon Counting" mode (PC). The events of these observations were first calibrated and screened using xrtpipeline v0.13.2. The applied Telescope Definition File (teldef) was based on XRT 
CALDB release 2013-06-01. After having extracted an image of the observation using xselect, we created a circular source region with a radius of 47 " corresponding to $90 \%$ of the PSF. In WT mode the circular background region of the same radius was selected at the edge of the one-dimensional image. During epoch 3 the count rate was, unfortunately, higher than 0.5 counts $\mathrm{s}^{-1}$, the nominal limit for pile-up since the outburst did not decay into quiescence as expected (see Fig. 1). To avoid pile-up in PC mode we compared the count rate profile with the expected PSF. We checked our results with the pileest tool, since the bad CCD columns due to a micro-meteorite hit might affect the count rate profile. The final source extraction region is an annulus with an outer radius of $47^{\prime \prime}$ and an inner radius of $22^{\prime \prime}$. The background region for epoch 3 is an annulus centered at the source position with an outer radius of $550^{\prime \prime}$ and an inner radius of $250^{\prime \prime}$.

The XRT-spectra were extracted using xselect. We only considered single events (grade 0 ) in the observations performed in PC mode (epoch 3). For the remaining observations in WT mode we also considered split events (grades $0-2$ ). The spectra for each Good Time Interval (GTI) in WT mode were added into a final single spectrum. The ARF was generated by xrtmkarf taking the effective area and filter transmission into account and correcting for vignetting and PSF.

We rebinned the Swift-XRT spectra to a SNR of 12 for epochs $1 \mathrm{~b}$ and 4 , and to 6 for epoch 3 , in order to retain enough spectral resolution around the iron line. These spectra were fitted in the $1-8 \mathrm{keV}$ range.

\section{Spectral analysis}

The spectral analysis was performed using the Interactive Spectral Interpretation System (ISIS, Houck \& Denicola 2000) v1.6.2-30. Unless stated otherwise, all uncertainties are given at the $90 \%$ confidence level and represent single parameter uncertainties $\left(\Delta \chi^{2}=2.71\right)$.

As found in our previous work (K13), the RXTE broad-band spectra of GRO J1008-57 can be well described by a powerlaw with an exponential cutoff and an additional black body. This model provides very good fits over more than two orders of magnitude in the source's flux. We applied the same model to the recent observations summarized in Table 1 in order to check and extend the covered range of fluxes. In K13 we found the temperature of the black body $k T=1.833 \pm 0.019 \mathrm{keV}^{1}$ and the folding energy of the cutoff power-law $E_{\text {fold }}=15.9 \pm 0.3 \mathrm{keV}^{1}$ to be independent of the source's flux and consistent among the outbursts. Furthermore, the bolometric flux of the black body, $F_{\mathrm{BB}}$, and the photon index of the power-law, $\Gamma$, were found to be well-defined functions of the source's $15-50 \mathrm{keV}$ flux, $F_{\mathrm{PL}}$. When checking these earlier results against the recent data analyzed here, we did not apply any of these findings a priori to the following spectral analysis.

We modeled the absorption by the interstellar medium (ISM) using TBnew ${ }^{2}$, a revised version of the model described

\footnotetext{
1 The uncertainties of some of the flux-independent parameters listed in Table 4 of K13 have been accidentally given on a confidence level slightly smaller than the stated $90 \%$. The affected parameters with correct uncertainties are the black body temperature $k T=1.833 \pm$ $0.019 \mathrm{keV}$, the folding energy $E_{\text {fold }}=15.9 \pm 0.3 \mathrm{keV}$, the flux of the galactic ridge $X$-ray emission $F_{3-10 \mathrm{keV}}=4.25 \pm 0.23 \times 10^{-12} \mathrm{erg} \mathrm{s}^{-1} \mathrm{~cm}^{-2}$ and its iron line parameters $E=6.35 \pm 0.03 \mathrm{keV}, \sigma=0.53 \pm 0.06 \mathrm{keV}$, and $F=2.39 \pm 0.18 \times 10^{-4}$ photons s ${ }^{-1} \mathrm{~cm}^{-2}$.

2 http://pulsar.sternwarte.uni-erlangen.de/wilms/ research/tbabs/
}

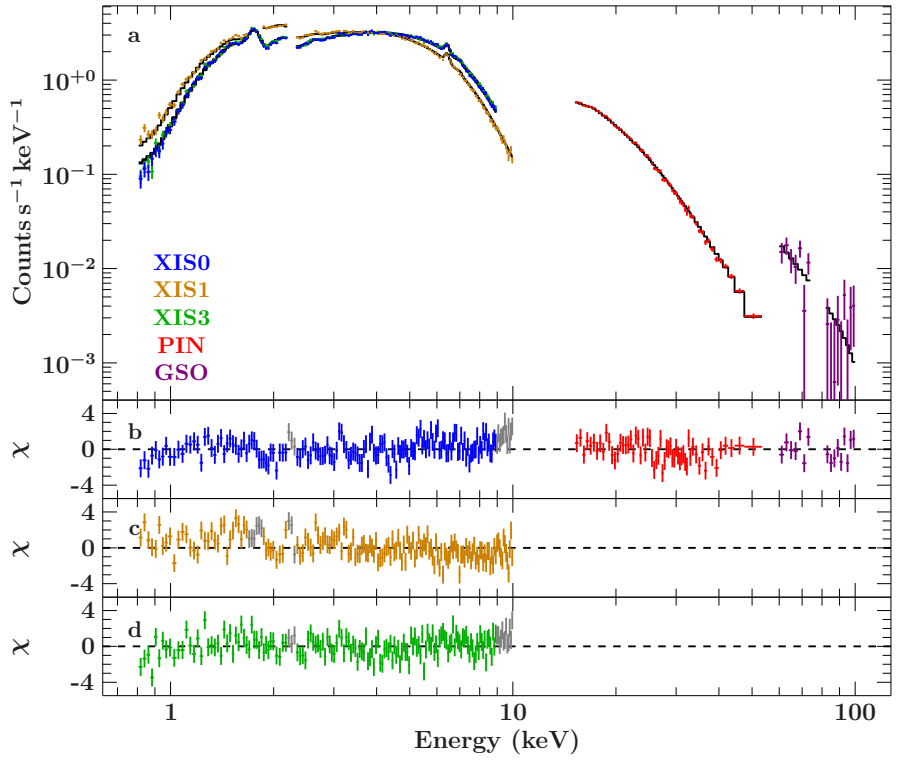

Fig. 2. a) The Suzaku-XIS (0: blue; 1: yellow; 3: green), -PIN (red), and -GSO (purple) spectra of the 2014 January observation of GRO J1008-57 (epoch 2) together with the best-fit model (black). $b-d$ ) The residuals to the best-fit model ( $b$ : XIS0, PIN, and GSO; $c$ : XIS1; $d$ : XIS3) showing the ignored energy bins due to calibration uncertainties (gray). The residuals of XIS1 $(c)$ are shown without taking the photon index shift, $\Delta \Gamma$, into account.

by Wilms et al. (2000). Element abundances were set to Wilms et al. (2000) and cross-sections were taken from Verner et al. (1996). Furthermore, GRO J1008-57 shows fluorescent emission of iron at $6.4 \mathrm{keV}$ and a CRSF around $78 \mathrm{keV}$, which we described by a Gaussian and a pseudo-Lorentzian absorption profile (CYCLABS, Makishima et al. 1990), respectively. Finally, we accounted for uncertainties in the flux calibration using a multiplicative constant, $c_{x}$, for each instrument.

\subsection{Suzaku (epoch 1a and 2)}

For the analysis of the Suzaku spectra, all broad-band continuum parameters were allowed to vary, which are the black body temperature, $k T$, the folding energy, $E_{\text {fold }}$, the power-law photon index, $\Gamma$, the black body flux, $F_{\mathrm{BB}}$, and the overall flux, $F_{\mathrm{PL}}$, in the $15-50 \mathrm{keV}$ range. The absorption column density, $N_{\mathrm{H}}$, the energy of the iron $\mathrm{K} \alpha$ line, $E_{\mathrm{Fe} \alpha}$, and its flux, $F_{\mathrm{Fe} \mathrm{K} \alpha}$, were free parameters as well. The width of the iron line was kept narrow, that is, fixed to $\sigma_{\mathrm{Fe} \mathrm{K} \alpha}=10^{-6} \mathrm{keV}$. From the best-fit we derived the iron line equivalent width, $\mathrm{EW}_{\mathrm{Fe} \mathrm{K} \alpha}$. The flux calibration constants, $c_{\mathrm{XIS} 0}, c_{\mathrm{XIS} 1}, c_{\mathrm{PIN}}$, and $c_{\mathrm{GSO}}$ were determined relative to $c_{\text {XIS3 }}=1$ (fixed). We note that during epoch $1 \mathrm{a}$ and 2 further calibration features were included in the fit as well (see Sect. 2.1). The CRSF was included in modeling epoch 1 only as it is not detected in epoch 2 (see the following for details).

A first fit to the data of the 2014 January outburst (epoch 2) results in a very good description of the data. We found, however, a line-like feature around $7 \mathrm{keV}$ that could be modeled by adding a second narrow Gaussian component. The fitted line energy of $E_{\mathrm{FeK} \beta}=6.85_{-0.09}^{+0.21} \mathrm{keV}$ and relative flux $F_{\mathrm{FeK} \beta} / F_{\mathrm{FeK} \alpha}=0.19_{-0.10}^{+0.10}$ are in excellent agreement with fluorescent $\mathrm{K} \beta$ emission from neutral iron. The Suzaku spectrum together with the final best-fit model is shown in Fig. 2. The corresponding fit parameters are listed in Table 2. 
M. Kühnel et al.: Evidence for different accretion regimes in GRO J1008-57

Table 2. Best-fit parameters of the Suzaku and simultaneous NuSTAR Swift spectra analyzed here.

\begin{tabular}{|c|c|c|c|c|c|}
\hline Parameter & $\begin{array}{l}\text { Epoch 1a } \\
\text { Suzaku }\end{array}$ & $\begin{array}{l}\text { Epoch 1b } \\
\text { NuSTAR-Swift }\end{array}$ & $\begin{array}{l}\text { Epoch } 2 \\
\text { Suzaku }\end{array}$ & $\begin{array}{l}\text { Epoch } 3 \\
\text { NuSTAR-Swift }\end{array}$ & $\begin{array}{l}\text { Epoch } 4 \\
\text { NuSTAR-Swift }\end{array}$ \\
\hline$\chi_{\text {red }}^{2} /$ d.o.f. & $1.224 / 579$ & $1.180 / 1808$ & $1.138 / 525$ & $0.956 / 579$ & $1.175 / 1796$ \\
\hline$F_{\mathrm{PL}}\left(10^{-9} \mathrm{erg} \mathrm{s}^{-1} \mathrm{~cm}^{-2}[15-50 \mathrm{keV}]\right)$ & $10.9_{-0.9}^{+0.9}$ & $10.357_{-0.024}^{+0.024}$ & $1.49_{-0.08}^{+0.08}$ & $0.3070_{-0.0030}^{+0.0030}$ & $7.121_{-0.018}^{+0.018}$ \\
\hline$\Gamma$ & $0.32_{-0.25}^{+0.16}$ & $0.476_{-0.025}^{+0.024}$ & $0.63_{-0.05}^{+0.05}$ & $1.15_{-0.08}^{+0.08}$ & $0.582_{-0.021}^{+0.020}$ \\
\hline$E_{\text {fold }}(\mathrm{keV})$ & $14.3_{-1.9}^{+1.8}$ & $14.86_{-0.24}^{+0.25}$ & $15.9_{-0.8}^{+0.08}$ & $19.6_{-1.4}^{+1.50}$ & $15.75_{-0.25}^{+0.26}$ \\
\hline$F_{\mathrm{BB}}\left(10^{-9} \mathrm{erg} \mathrm{s}^{-1} \mathrm{~cm}^{-2}\right)$ & $1.2_{-0.4}^{+0.5}$ & $0.90_{-0.08}^{+0.08}$ & $0.54_{-0.04}^{+0.8}$ & $0.063_{-0.010}^{+-0.4011}$ & $0.82_{-0.05}^{+0.05}$ \\
\hline$k T(\mathrm{keV})$ & $1.89_{-0.22}^{+0.22}$ & $1.689_{-0.021}^{+0.023}$ & $1.90_{-0.05}^{+0.04}$ & $1.69_{-0.07}^{+0.08}$ & $1.949_{-0.027}^{+0.029}$ \\
\hline$k T_{2}(\mathrm{keV})$ & $0.489_{-0.033}^{-0.22}$ & $-\quad-0.021$ & $-\quad-0.05$ & - & $-\quad-0.027$ \\
\hline$F_{\mathrm{BB} 2} / F_{\mathrm{BB}} *$ & $0.46_{-0.09}^{+0.12}$ & - & - & - & - \\
\hline$N_{\mathrm{H}}\left(10^{22} \mathrm{~cm}^{-2}\right)$ & $1.45_{-0.07}^{+0.07}$ & $1.44_{-0.05}^{+0.05}$ & $1.60_{-0.04}^{+0.04}$ & $4.0_{-0.7}^{+0.7}$ & $1.66_{-0.05}^{+0.05}$ \\
\hline$E_{\mathrm{Fe} \mathrm{K} \alpha}(\mathrm{keV})$ & $6.405_{-0.010}^{+0.030}$ & $6.528_{-0.014}^{+0.014}$ & $6.433_{-0.021}^{+0.010}$ & $6.43_{-0.04}^{+0.06}$ & $6.559_{-0.014}^{+0.014}$ \\
\hline$\sigma_{\mathrm{Fe} \mathrm{K} \alpha}(\mathrm{keV})$ & $10^{-6 \dagger}$ & $0.306_{-0.019}^{-0.014}$ & $10^{-6 \dagger^{-0.021}}$ & $10^{-6 \dagger}$ & $0.292_{-0.018}^{+0.019}$ \\
\hline$F_{\mathrm{Fe} \mathrm{K} \alpha}\left(10^{-4} \mathrm{ph} \mathrm{s}^{-1} \mathrm{~cm}^{-2}\right)$ & $26.7_{-3.0}^{+3.0}$ & $73_{-4}^{+4^{-0.019}}$ & $9.1_{-0.8}^{+0.8}$ & $1.24_{-0.27}^{+0.27}$ & $53.1_{-2.5}^{+2.6}$ \\
\hline$E W_{\mathrm{Fe} \mathrm{K} \alpha}(\mathrm{eV})$ & $36_{-5}^{+5}$ & $102_{-5}^{+5}$ & $50_{-5}^{+5}$ & $26_{-4}^{+4}$ & $90_{-5}^{+5}$ \\
\hline$E_{\mathrm{Fe} \mathrm{K} \beta}(\mathrm{keV})$ & 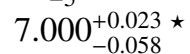 & - & $6.85_{-0.09}^{+0.21}$ & - & - \\
\hline$F_{\mathrm{Fe} \mathrm{K} \beta}\left(10^{-4} \mathrm{ph} \mathrm{s}^{-1} \mathrm{~cm}^{-2}\right)$ & $11.1_{-3.0}^{+3.0 \star} \star$ & - & $1.6_{-0.8}^{+0.89}$ & - & - \\
\hline$E W_{\mathrm{Fe} \mathrm{K} \beta}(\mathrm{eV})$ & $16_{-5}^{+5 \star}$ & - & $10_{-5}^{+5}$ & - & - \\
\hline$E_{\mathrm{FexxIV}}(\mathrm{keV})$ & $6.689_{-0.022}^{+0.018}$ & - & - & - & - \\
\hline$F_{\mathrm{FexxIV}}\left(10^{-4} \mathrm{ph} \mathrm{s}^{-1} \mathrm{~cm}^{-2}\right)$ & $23.6_{-3.0}^{+3.0}$ & - & - & - & - \\
\hline$E W_{\mathrm{Fe} \text { xxiv }}(\mathrm{eV})$ & $33_{-5}^{+5}$ & - & - & - & - \\
\hline$E_{\text {сус }}(\mathrm{keV})$ & $75.9_{-1.4}^{+1.6}$ & $70.7_{-1.5}^{+1.7}$ & - & - & $70.4_{-1.9}^{+2.7}$ \\
\hline$W_{\text {cyc }}(\mathrm{keV})$ & $10^{\dagger}$ & $10^{\dagger}$ & - & - & $10^{\dagger}$ \\
\hline$\tau_{\mathrm{cyc}}$ & $1.8_{-0.5}^{+0.5}$ & $0.99_{-0.13}^{+0.18}$ & - & - & $0.75_{-0.14}^{+0.24}$ \\
\hline$c_{\mathrm{XIS} 0} / c_{\mathrm{XIS} 3}$ & $0.980_{-0.005}^{+0.005}$ & - & $0.943_{-0.006}^{+0.006}$ & - & - \\
\hline$c_{\mathrm{XIS} 1} / c_{\mathrm{XIS} 3}$ & $1.046_{-0.005}^{+0.005}$ & - & $0.869_{-0.012}^{+0.012}$ & - & - \\
\hline$c_{\mathrm{PIN}} / c_{\mathrm{XIS} 3}$ & $1.38_{-0.11}^{+0.12}$ & - & $1.31_{-0.07}^{+0.07}$ & - & - \\
\hline$c_{\mathrm{GSO}} / c_{\mathrm{XIS} 3}$ & $1.43_{-0.13}^{+0.15}$ & - & $1.20_{-0.22}^{+0.23}$ & - & - \\
\hline$c_{\mathrm{FPMB}} / c_{\mathrm{FPMA}}$ & - & $1.0269_{-0.0015}^{+0.0015}$ & - & $1.034_{-0.005}^{+0.005}$ & $1.0147_{-0.0015}^{+0.0015}$ \\
\hline$c_{\mathrm{XRT}} / c_{\mathrm{FPMA}}$ & - & $1.187_{-0.009}^{+0.009}$ & - & $1.00_{-0.08}^{+0.08}$ & $1.233_{-0.010}^{+0.010}$ \\
\hline
\end{tabular}

Notes. The uncertainties are given at the $90 \%$ confidence level. The parameters regarding the calibration of the XISs and PIN are given in Sect. 3.1. ${ }_{(\ddagger)}^{(\ddagger)}$ Ratio between the bolometric fluxes of the black bodies. ${ }^{(\star)}$ Epoch 1a is a blend of FeK $\beta$ and FeK $\alpha$ XXV. ${ }^{(\dagger)}$ Fixed.

The Suzaku spectrum during the giant 2012 November outburst (epoch 1a) showed even more features. After applying the same K13 model as for epoch 2, strong residuals in absorption in PIN and GSO at energies above $60 \mathrm{keV}$ remained, which we attributed to the high-energy CRSF (Bellm et al. 2014; Yamamoto et al. 2013, 2014). Adding such a feature with its width, $W_{\text {cyc }}$, fixed to $10 \mathrm{keV}$ improved the fit significantly $\left(\Delta \chi^{2}=\right.$ $353)$. The energy and depth of the CRSF, $E_{\text {cyc }}$ and $\tau_{\text {cyc }}$, respectively, were consistent with those found earlier. The XIS-spectra showed an excess towards lower energies, which could be described with a second black body at $k T_{2}=0.489_{-0.033}^{+0.028} \mathrm{keV}$ and improved the fit further $\left(\Delta \chi^{2}=456\right)$. Finally, the emission lines of iron at $6.40,6.67$, and $7.00 \mathrm{keV}$, which we detected in our earlier analysis of this dataset (K13), were again detected. The line at $6.67 \mathrm{keV}$ represents $\mathrm{K} \alpha$ emission from He-like iron (Fe XXIV), while the line at $7.00 \mathrm{keV}$ is a combination of the $\mathrm{K} \beta$ from neutral iron and $\mathrm{K} \alpha$ from $\mathrm{H}$-like iron (Fe XXV). The total flux in the fitted line was significantly higher than what is expected from neutral $\mathrm{K} \beta$ alone. The fit parameters of the final best-fit are listed in Table 2. For a figure showing the full Suzaku spectrum see
Bellm et al. (2014, Fig. 4) and K13, Fig. 15, for a close-up of the iron line region.

\subsection{NuSTAR and Swift (epochs 1b, 3, and 4)}

In a first step, we applied again the K13 model to the NuSTAR and Swift spectra (see Sect. 3.1). The broad-band continuum parameters were kept free as for the Suzaku analysis above. We added a Gaussian component in order to model the apparent neutral iron line with its centroid energy, $E_{\mathrm{Fe} \mathrm{K} \alpha}$, its flux and equivalent width, $F_{\mathrm{Fe} \mathrm{K} \alpha}$ and $E W_{\mathrm{Fe} \mathrm{K} \alpha}$, respectively, and its width, which we fixed to $\sigma_{\mathrm{Fe} \mathrm{K} \alpha}=10^{-6} \mathrm{keV}$. Flux calibration constants, $c_{\mathrm{FPMB}}$ and $c_{\mathrm{XRT}}$, relative to $c_{\mathrm{FPMA}}=1$ were included during the fit.

This model was able to describe the spectra taken during epoch 3, when GRO J1008-57's luminosity was just above the detection limit of Swift-BAT (compare Fig. 1). The spectra during high luminosities of the source (epochs $1 \mathrm{~b}$ and 4 ) could not, however, be fitted well with this model. There were clear indications for the CRSF towards the higher end of NuSTAR's energy range. Including a CRSF with its width fixed to $W_{\text {cyc }}=10 \mathrm{keV}$ 


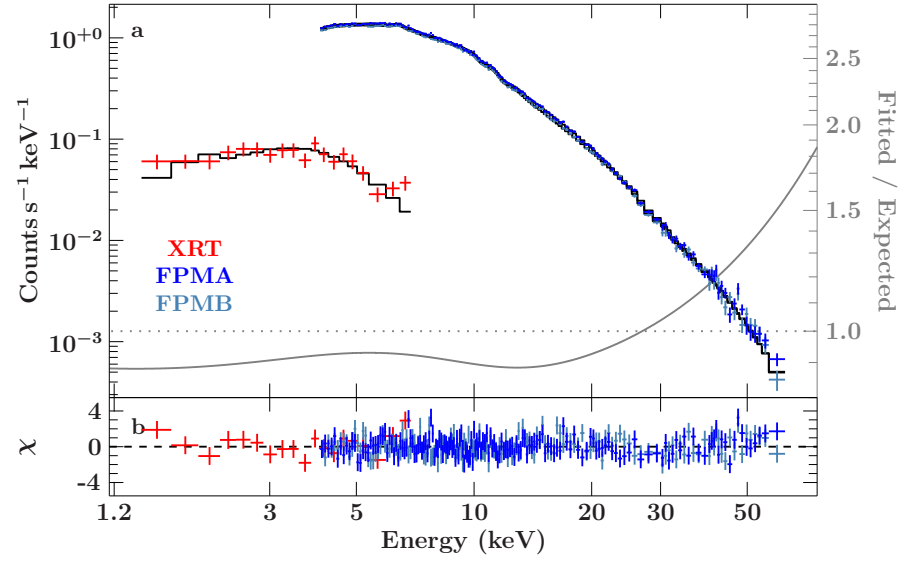

Fig. 3. a) NuSTAR-FPMA (dark blue), -FPMB (light blue), and SwiftXRT spectrum (red) of GRO J1008-57 in 2014 December after the second peak of its triple peaked outburst (epoch 3). The best-fit model is shown in black and the gray line shows its ratio to the spectral shape as predicted by our spectral evolution model (see Sect. 5 and Fig. 6). The data have been rebinned for display purposes. $b$ ) The residuals of the best-fit model.

as for the Suzaku data (see Sect. 3.1) led to a very good description of the spectra $\left(\Delta \chi^{2}=527\right.$ for epoch $1 \mathrm{~b}$ and $\Delta \chi^{2}=286$ for epoch 4). Slight residuals around the neutral iron line remained in all epochs. The energy resolution of the NUSTAR FPMs, however, does not allow resolution of further emission lines, such as iron $\mathrm{K} \beta$ or ionized iron, as was possible with the Suzaku-XISs. Nevertheless, varying the width of the iron $\mathrm{K} \alpha$ line, $\sigma_{\mathrm{Fe} \mathrm{K} \alpha}$, was sufficient to get rid of these residuals. The best-fit energy, $E_{\mathrm{Fe} \mathrm{K} \alpha}$, was higher than the $6.4 \mathrm{keV}$ expected from neutral iron. Together with a width of a few $100 \mathrm{eV}$ these results point towards a blend of several emission lines, possibly from ionized iron as has been detected in the Suzaku data (see Sect. 3.1). For epoch 1b, the flux in the line of around $75 \mathrm{ph} \mathrm{s}^{-1} \mathrm{~cm}^{-2}$ agrees with the sum of the fluxes in the individual lines resolved in the Suzaku-XIS spectra (epoch 1a). The final fit-parameters for all epochs of $N u S$ TAR and Swift observations are listed in Table 2. The spectra and corresponding models for epochs 3 and 4 are shown in Figs. 3 and 4, respectively. For epoch $1 \mathrm{~b}$ see Bellm et al. (2014, Fig. 3). We note that the line-like residuals in Fig. $4 \mathrm{~b}$ at $\sim 1.8 \mathrm{keV}$ and $\sim 2.2 \mathrm{keV}$ are only visible due to the coarser channel binning used in this figure. These features are calibration uncertainties in Swift-XRT around the Si K edge and the Au M edge, respectively (Godet et al. 2007; Hurkett et al. 2008). Due to their low significance they do not have any effect on the spectral parameters here.

\section{Discussion on individual observations}

We have successfully applied the model used in our previous study of GRO J1008-57 (K13) to recent Suzaku and simultaneous NuSTAR and Swift spectra. We compare the results of K13, especially the parameter evolution with the X-ray flux, with the results of the spectral analysis presented here in Sect. 5 separately. In this section we discuss our results in their individual context.

\subsection{CRSF and 2nd black body}

During the giant 2012 November outburst we can confirm the presence of a CRSF in the Suzaku and NuSTAR data (epochs 1a and 1b). Furthermore, we have detected the CRSF in the NuSTAR data during epoch 4 , which happened at a source luminosity level

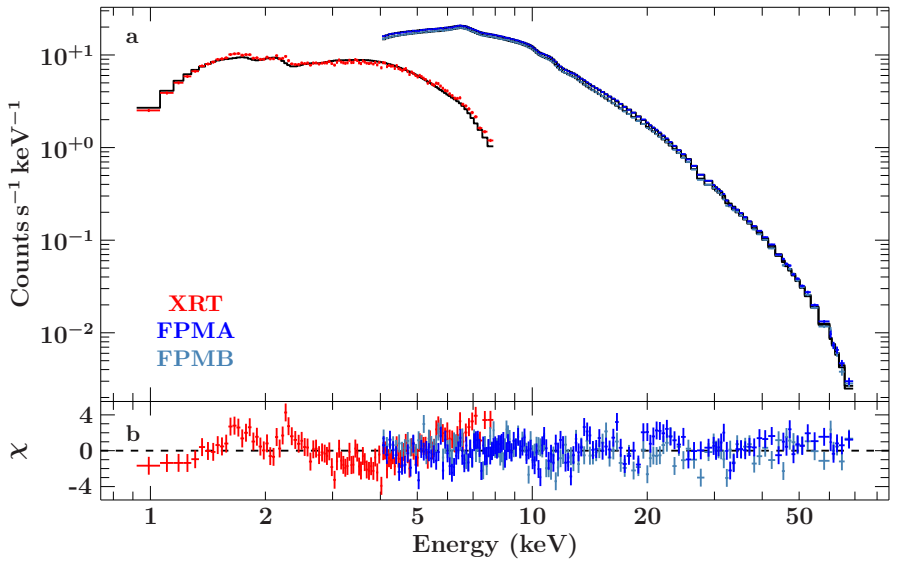

Fig. 4. a) NuSTAR-FPMA (dark blue), -FPMB (light blue), and SwiftXRT spectrum (red) of GRO J1008-57 in 2015 January shortly after maximum flux of the third peak of the triple peaked outburst (epoch 4). The black line shows the best-fit model. The data have been rebinned for display purposes. $b$ ) The residuals of the best-fit model.

comparable to that during epochs $1 \mathrm{a}$ and $1 \mathrm{~b}$. There is no hint of a cyclotron line in the NUSTAR data during epoch 3 and in the Suzaku data during epoch 2, when the source was at a much lower luminosity level. We cannot, however, exclude its presence due to the lower statistic. In the Suzaku data of the giant outburst (epoch 1a), we find the cyclotron line energy $E_{\text {cyc }}=75.9_{-1.4}^{+1.6} \mathrm{keV}$ to be in excellent agreement with $75.5_{-1.5}^{+2.5} \mathrm{keV}$ and $78_{-2}^{+3} \mathrm{keV}$ as found in the earlier analyses of these data by Yamamoto et al. (2013, 2014) and Bellm et al. (2014), respectively. The apparent difference in the measured CRSF energies between NuSTAR (epoch $1 \mathrm{~b}$ and 4) and Suzaku (epoch 1a) is likely due to systematic uncertainties in this energy range, which is at the upper end of the useful energy range for both instruments. Furthermore, a slight change in the continuum parameters, especially the photon index, $\Gamma$, or the folding energy, $E_{\mathrm{fold}}$, influences the CRSF parameters at this high energy. Thus, we cannot draw definite conclusions from the apparent difference between the Suzaku (epoch 1a) and the NUSTAR results (epochs $1 \mathrm{~b}$ and 4).

In the Suzaku data of the giant outburst of GRO J1008-57 in 2012 (epoch 1a) a second black body with $k T_{2}=0.489_{-0.033}^{+0.028} \mathrm{keV}$ is required to fit the low-energy part of the XIS-spectra successfully. We had already detected this feature in our earlier analysis of these data together with Swift spectra (K13). On the other hand, the feature was not detected in the two Suzaku observations at lower luminosities (epoch 2 and during the decay of the 2007 data, see K13 for details) as well as in the Swift data during the maximum of the 2007 outburst (see K13). Thus, it is likely that the soft part of GRO J1008-57's spectrum at $\lesssim 3 \mathrm{keV}$ changed during the giant outburst. In combination with the evolution of the other spectral parameters with flux, as presented Sect. 5, this is evidence for the existence of different accretion regimes in GRO J1008-57. Bellm et al. (2014), who used the NPEX model for the broad-band continuum, found this second black body at the same temperature as we did, while they did not detect it in the NUSTAR data alone. We found that this feature is not significantly detected in the combined NuSTAR and Swift data as well. The broad-band continuum parameters agree, however, between Suzaku and NuSTAR. Thus, the presence of this feature does not significantly influence the remaining spectral parameters. Adding this feature to the $N U S T A R$ spectrum at a fixed temperature indeed leads to consistent results with the Suzaku spectrum. 


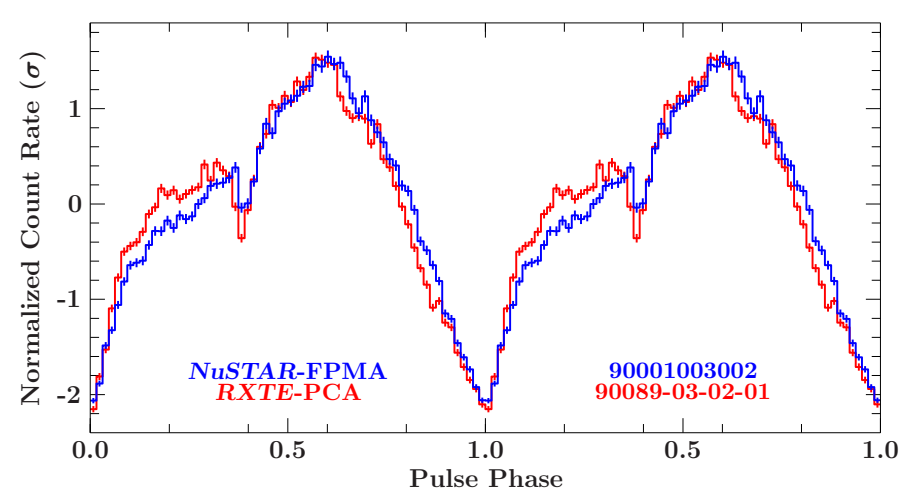

Fig. 5. Pulse profiles of GRO J1008-57 during epoch 3 (blue; NuSTARFPMA) and 2005 (red; RXTE-PCA). The RXTE observation was performed at a similar flux level of the source compared to the NuSTAR observation. The pulse profiles have been normalized to a mean count rate of zero and a standard deviation, $\sigma$, of unity. Pulse phase zero is defined as the minimum count rate.

\subsection{Spectral anomaly of epoch 3}

During the transition from the second to the third outburst (epoch 3) during GRO J1008-57's “triple-peaked" outburst, the spectral parameters disagree with the correlation between spectral parameters as found in the other observations of this source (see Sect. 5 for details). In particular, the folding energy, $E_{\text {fold }}$, of $\sim 19.6 \mathrm{keV}$ is significantly higher than the $15-16 \mathrm{keV}$ as found during all other epochs (see Table 2). The gray line in Fig. 3a shows the ratio between the best-fit model spectrum and the predicted spectrum assuming the measured source flux at 15$50 \mathrm{keV}$ as listed in Table 2. As can be seen, this flux ratio increases exponentially above $\sim 30 \mathrm{keV}$ up to a factor of 2 around $70 \mathrm{keV}$. These facts still hold true if the NUSTAR and Swift spectra are fitted with taking the CRSF into account, that is, the presence or absence of the CRSF has no influence on the model parameters.

To draw conclusions about the reason for this spectral anomaly we compared the pulse profile of the corresponding NuSTAR observation with an RXTE observation in 2005 at a similar flux level (ObsID 90089-03-02-01). As RXTE-PCA and NuSTAR-FPMA are sensitive over comparable energy ranges (3-60 and 3-78 keV), differences in the pulse profiles can be associated with changes in the accretion geometry. After correcting the light curves for binary motion using the orbital parameter listed in K13, we folded the NUSTAR and RXTE light curves on 93.445 s and $93.675 \mathrm{~s}$, respectively, which correspond to the spin periods of GRO J1008-57 and were determined from the data using the epoch folding technique (see, e.g., Leahy et al. 1983).

The resulting pulse profiles shown in Fig. 5 were normalized to a mean count rate of zero and a standard deviation of unity (see, e.g., Ferrigno et al. 2011; Schönherr et al. 2014). Both pulse profiles are very similar in shape. We therefore concluded that the accretion geometry between these observations was very similar as well, which hints at an origin outside of the neutron star's magnetosphere for the spectral anomaly. The observed changes in the pulse profile of A0535+26 during pre-outburst flares (Caballero et al. 2008) were explained by Postnov et al. (2008) as the result of magnetospheric instabilities. Since the pulse profile of GRO J1008-57 during the flaring epoch 3 is consistent with the expected pulse profile shape, it is unlikely that these instabilities are the origin for the observed flaring activity.

\section{3. "Triple-peaked" outburst morphology}

After the first confirmed giant outburst of GRO J1008-57 in 2012 November (epochs 1a and 1b) since its discovery in 1993 , the source featured an expected type I outburst in January 2014 (epoch 2). Surprisingly, after the type-I outburst in September 2014, GRO J1008-57 went into a type-II outburst again. But instead of fading into quiescence after this second outburst (epoch 3), the source showed a third outburst in a single orbit (epoch 4). In fact, the peak of the third outburst was reached at apastron (see Fig. 1 and Kretschmar et al. 2015).

Figure 1 shows the light curve morphology of GRO J1008-57 during this "triple-peaked" outburst in 2014/2015, which lasted for half the orbital period of $\sim 250 \mathrm{~d}$. The transition between the first two outbursts occurred rather smoothly, which has also been observed during the known "doublepeaked" outbursts of A 0535+262 (Caballero et al. 2013, Fig. 1) and GX 304-1 (Postnov et al. 2015b, Fig. 1). The peak separation in GRO J1008-57 of $\sim 65 \mathrm{~d}=0.25 P_{\text {orb }}$ is, however, much larger than in these systems, which show separations of $\sim 9 \mathrm{~d}=0.08 P_{\text {orb }}$ with $P_{\text {orb }}=111.1 \mathrm{~d}$ in the case of A $0535+26$ (Finger et al. 2006) and $\sim 25 \mathrm{~d}=0.19 P_{\text {orb }}$ with $P_{\text {orb }}=132.5 \mathrm{~d}$ in the case of GX 304-1 (Priedhorsky \& Terrell 1983). As argued by Postnov et al. (2015b), the "double-peaked" outburst of GX 304-1 is due to a misaligned Be-disk with respect to the orbital plane. Once the disk has grown sufficiently beyond the distance between the stellar surface and the neutron star's orbit a second intersection might be possible. This is consistent with recent theoretical investigations by Okazaki et al. (2013). The geometries of the Be-disk and the orbit are unique for each BeXRB, although there are similarities among this class. Thus, a difference of the peak separation up to a factor of $\sim 3$ in orbital phase is not astonishing. We note that the outbursts of XTE J1946+274 occurred twice per orbital period $\left(P_{\text {orb }} \sim 172 \mathrm{~d}\right.$; see, e.g., Marcu-Cheatham et al. 2015, and references therein), that is, the peak separation in this system is about 0.5 in orbital phase. As the system shows $\geq 5$ outbursts in a row, this morphology is, however, different compared to the "double-peaked" outbursts.

Between the second and third peak of GRO J1008-57's "triple-peaked" outburst, the light curve is not as smooth as the transition between the first two peaks. Rather, the source stayed at a more or less constant level for $\sim 50 \mathrm{~d}$ with a weak flaring activity. A similar behavior was also observed before the giant 2012 November outburst (see Fig. 1), when the flaring activity was, however, much stronger and lasted for $\sim 60 \mathrm{~d}$. These flares occurred quasi-periodically with a period of around $9 \mathrm{~d}$. A speculative explanation for this different behavior compared to the smooth transition might be external torques onto the accretion disk as studied by Doğan et al. (2015). They argue that for an accretion disk of a certain size and inclined with respect to the orbital plane, the torques might overpower the internal disk torques. Consequently, the disk would break up into slices, which would precess independently of each other. Doğan et al. (2015) thus conclude that the mass accretion rate onto the compact object gets modulated. In their example simulation (see Fig. 5 of Doğan et al. 2015) the period of this modulation is much longer than the orbital period, which makes it difficult to reconcile with the observed $9 \mathrm{~d}$ period.

\section{Parameter evolution with flux}

In K13 we found that the broad-band continuum of GRO J1008-57 is a function of the overall X-ray flux. The black 
body temperature, $k T$, and the folding energy, $E_{\text {fold }}$, are independent of the $15-50 \mathrm{keV}$ flux, $F_{\mathrm{PL}}$, and consistent among the outbursts. The power-law photon index, $\Gamma$, and the black body flux, $F_{\mathrm{BB}}$, show a tight correlation with $F_{\mathrm{PL}}$. These results are mainly based on observations taken by RXTE with the addition of one Swift and one Suzaku pointing.

The black body temperature found by analyzing the new Suzaku (epochs 1a and 2) and joint NuSTAR and Swift observations (epochs $1 \mathrm{~b}, 3$, and 4) are all within $0.15 \mathrm{keV}$ of $k T=$ $1.833 \pm 0.019 \mathrm{keV}$ as found by K13. Despite this small temperature range, the values during the bright $N U S T A R$ observations (epochs 1b and 4) are, however, significantly lower than the mean value. The folding energy of $E_{\text {fold }}=15.92_{-0.30}^{+0.29} \mathrm{keV}$ that we measured previously in K13 is consistent with the observations analyzed here with the exception of the NuSTAR epochs $1 \mathrm{~b}$ and 3. While GRO J1008-57's spectral anomaly during epoch 3 has been discussed in Sect. $4.2, E_{\text {fold }}$ during epoch $1 \mathrm{~b}$ is $\sim 1 \mathrm{keV}$ lower compared to the RXTE result of K13. In order to understand these apparent differences in $k T$ and $E_{\text {fold }}$ we investigated contour maps of these parameters against other continuum parameters. We discovered parameter degeneracies, especially between the photon index, $\Gamma$, and the folding energy, $E_{\text {fold }}$. In fact, the folding energy found previously by K13 is within $2.7 \sigma$ (for two degrees of freedom) of the best-fit of epoch $1 \mathrm{~b}$ as listed in Table 2. Fixing $E_{\text {fold }}=15.92 \mathrm{keV}$ indeed leads to a similar goodness of the fits for all epochs (except epoch 3 as discussed above). Thus, we conclude that the flux-independent parameters still seem to be the same for the newer data analyzed here (except epoch 3), which confirms our previous results (K13).

In $\mathrm{K} 13$ we also discovered that the photon index, $\Gamma$, and the black body flux, $F_{\mathrm{BB}}$, are functions of the overall $15-50 \mathrm{keV}$ source flux, $F_{\mathrm{PL}}$. In particular, the same behavior is found in all outbursts. Figure 6 is an updated version of our previous RXTE results (Fig. 10 of K13), where the best-fit $\Gamma$ and $F_{\mathrm{BB}}$ over $F_{\mathrm{PL}}$ of the newer data have been added (see Table 2). We note that since two fit parameters are plotted against each other in each panel, the uncertainties have to be calculated assuming two degrees of freedom. As we will fit these parameters below, we are providing $1 \sigma$ uncertainties $\left(\Delta \chi^{2}=2.30\right)$.

The parameters of the Suzaku epoch 2 (green triangle at $\sim 1.5 \times 10^{-9} \mathrm{erg} \mathrm{s}^{-1} \mathrm{~cm}^{-2}$ ) are consistent with the behavior seen earlier (black triangle). Furthermore, the NuSTAR and Suzaku observations during the giant 2012 outburst (the most luminous blue circle and green triangle) also give consistent results, although a second black body was necessary to describe the Suzaku data. Interestingly, the recent NuSTAR observation during the third outburst of the "triple-peaked" outburst (blue circle at $\sim 7 \times 10^{-9} \mathrm{erg} \mathrm{s}^{-1} \mathrm{~cm}^{-2}$ ) results in the same parameters as for the 2012 giant outburst, although the source was $\sim 45 \%$ brighter then. The data from epoch 3 (blue circle marked with 1), where the folding energy, $E_{\text {fold }}$, was not consistent with all the other observations (see Sect. 4.2 for a discussion), is almost consistent with the apparent parameter evolution. Due to the inconsistency in $E_{\text {fold }}$ we, however, ignore this observation until the end of this section.

In the following, we model the apparent evolution of the power-law photon index, $\Gamma$, and the black body flux, $F_{\mathrm{BB}}$, with the source flux, $F_{\mathrm{PL}}$, using phenomenological functions,

$\log : X\left(F_{\mathrm{PL}}\right)=a_{X}+b_{X} \log \left(F_{\mathrm{PL}} / 10^{-9} \mathrm{erg} \mathrm{s}^{-1} \mathrm{~cm}^{-2}\right)$, lin: $X\left(F_{\mathrm{PL}}\right)=b_{X}\left(F_{\mathrm{PL}} / 10^{-9} \mathrm{erg} \mathrm{s}^{-1} \mathrm{~cm}^{-2}-a_{X}\right)$,

pow: $X\left(F_{\mathrm{PL}}\right)=a_{X}\left(F_{\mathrm{PL}} / 10^{-9} \mathrm{erg} \mathrm{s}^{-1} \mathrm{~cm}^{-2}\right)^{b_{X}}$,

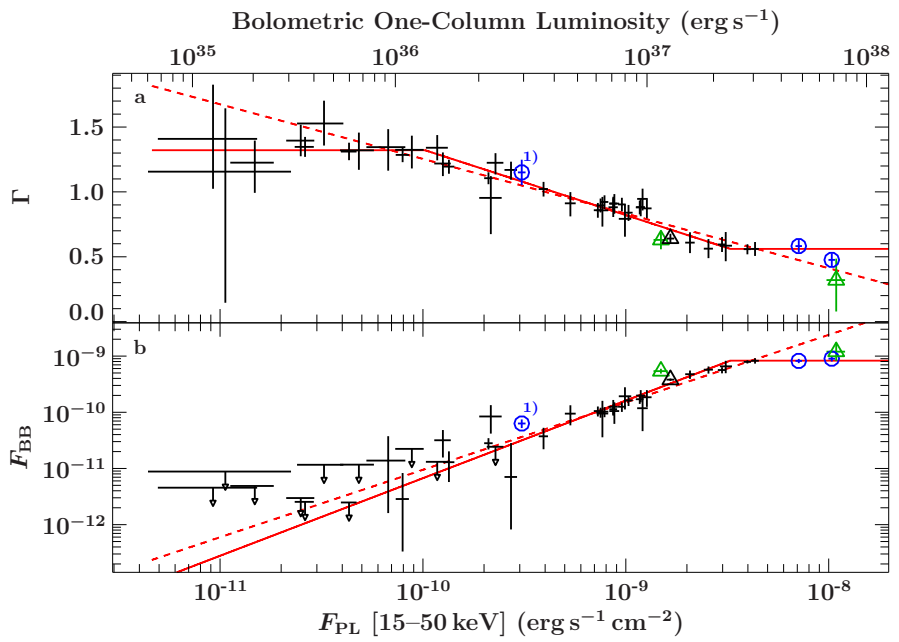

Fig. 6. Evolution of the spectral parameters with the $15-50 \mathrm{keV}$ flux, $F_{\mathrm{PL}}$, of GRO J1008-57. a) The power-law photon index, $\Gamma$, and $b$ ) the black body flux $F_{\mathrm{BB}}$ (in erg s $\mathrm{cm}^{-1}$ ), with the source's flux (uncertainties represent $1 \sigma$ uncertainties with two degrees of freedom). The black data points are the results of our previous RXTE and the black triangle the joined Suzaku RXTE analysis (K13). Their fluxes have been corrected for calibration uncertainties (see the text for details). The new results presented in this paper are the green triangles based on Suzaku and the blue circles based on NUSTAR data. The red lines show the empirical model with (solid) and without (dashed) the breaks at certain luminosity levels as described in the text. The NUSTAR results marked with 1) have been excluded from these fits. The bolometric luminosity for one accretion column has been calculated as described in Sect. 5.

where $X$ is either the photon index, $\Gamma$, or the black body flux, $F_{\mathrm{BB}}$. In the case of fitting the evolution of the black body flux, that is, $X=F_{\mathrm{BB}}$, we restrict the black body to be in emission only, that is, $F_{\mathrm{BB}}\left(F_{\mathrm{PL}}\right) \geq 0$ for all source fluxes, $F_{\mathrm{PL}}$. In order to fit a model consisting of these functions to one of the parameter evolutions shown in Fig. 6 we minimize the $\chi^{2}$ accounting for the asymmetric uncertainties in both the flux, $F_{\mathrm{PL}}$, and the parameter of interest, $X$.

During a first investigation of the parameter evolutions, we have ignored data at $F_{\mathrm{PL}}<8 \times 10^{-11} \mathrm{erg} \mathrm{s}^{-1} \mathrm{~cm}^{-2}$ in order to remove any possible bias introduced by the upper limits or large uncertainties at lower fluxes. Furthermore, we ignored data at $F_{\mathrm{PL}}>3 \times 10^{-9} \mathrm{erg} \mathrm{s}^{-1} \mathrm{~cm}^{-2}$ due to an insufficient description of the observed parameter evolutions at these high fluxes regardless of the chosen model. We model and discuss this discrepancy further below. We find that the photon index evolution, $\Gamma\left(F_{\mathrm{PL}}\right)$, is described best using a logarithmic function (log: $\chi^{2}=97.0$; pow: $\chi^{2}=183.1$; lin: $\chi^{2}=399.3$; all with 28 degrees of freedom). A power-law dependency fits the evolution of the black-body flux, $F_{\mathrm{BB}}\left(F_{\mathrm{PL}}\right)$, well (pow: $\chi^{2}=46.4$; lin: $\chi^{2}=69.1 ; \log : \chi^{2}=185.9$; all with 28 degrees of freedom).

Although the data follow these models well at first glance (see Fig. 6) the goodness of the fits as given above are not acceptable. A reduced $\chi^{2}$ near unity is, however, required for a reasonable interpretation of the resulting model parameters within their uncertainties. The reason for the large $\chi^{2}$ values is a few data points with very small uncertainties compared to the complete data set. These data points are, however, only a few percent off from the models. We have tried to fit these differences by introducing calibration constants similar to those during the spectral analysis (see Sect. 3). This approach failed due to the low number of data points for NuSTAR and Suzaku compared to RXTE and due to very similar spectral parameters among these 
Table 3. Parameters of the best-fitting combination of models describing the spectral evolution as shown in Fig. 6.

\begin{tabular}{ccc}
\hline \hline & $\begin{array}{c}\Gamma\left(F_{\mathrm{PL}}\right) \\
\text { dbl-brkn log }\end{array}$ & $\begin{array}{c}F_{\mathrm{BB}}\left(F_{\mathrm{PL}}\right) \\
\text { high-brkn pow }\end{array}$ \\
\hline$a_{X}$ & $0.821_{-0.019}^{+0.019}$ & $0.162_{-0.013}^{+0.013}$ \\
$b_{X}$ & $-0.51_{-0.05}^{+0.05}$ & $1.38_{-0.11}^{+0.12}$ \\
$F_{\text {brk,lo }}$ & $0.10_{-0.04}^{+0.05}$ & - \\
$F_{\text {brk,hi }}{ }^{a}$ & \multicolumn{2}{c}{$3.27_{-0.30}^{+0.33}$} \\
$\chi^{2 b}$ & 36.41 & 44.81 \\
$\chi_{\text {red }}^{2} /$ d.o.f. ${ }^{c}$ & \multicolumn{2}{c}{$0.97 / 84$} \\
\hline
\end{tabular}

Notes. The definitions of the models are given in the text. ${ }^{(a)} F_{\text {brk,hi }}$ has been determined by a joint fit to both correlations. ${ }^{(b)}$ The $\chi^{2}$ sum over all 45 data points for each parameter evolution. ${ }^{(c)}$ The total goodness of the fit.

missions at high luminosities of GRO J1008-57. To take into account the systematic effect of these offsets, we have added a systematic uncertainty of 0.03 in $\Gamma$ (corresponding to $2-7 \%$ relative uncertainty) and a relative uncertainty of $3.5 \%$ in both flux parameters, $F_{\mathrm{PL}}$ and $F_{\mathrm{BB}}$. These additional uncertainties are consistent with known energy and flux cross-calibration uncertainties between different X-ray missions and their instruments (see, e.g., Kirsch et al. 2005; Tsujimoto et al. 2011; or Madsen et al. 2017). We note that we cannot exclude source variability on a few percent level as a reason for the large $\chi^{2}$ values besides calibration uncertainties. This does not, however, affect any of the following conclusions given the overall change in $\Gamma$ and $F_{\mathrm{BB}}$ by a factor of 2-3 and by a few orders of magnitude, respectively.

When including all available data over the full flux range (with the exception of epoch 3), we find that the recent observations (when the source was at very high luminosities) are not consistent with a logarithmic function for $\Gamma\left(F_{\mathrm{PL}}\right)$ and, especially, a power-law function for $F_{\mathrm{BB}}\left(F_{\mathrm{PL}}\right)$ with a single slope. This is similar to what we found for lower fluxes in K14. For these three epochs (1a, 1b, and 4) we measure the same parameter values within their uncertainties, despite them being taken at very different fluxes. This behavior can be fitted much better by a flattening of the correlation towards higher fluxes, $F_{\text {brk }}$,

high-brkn: $X^{\prime}\left(F_{\mathrm{PL}}\right)= \begin{cases}X\left(F_{\mathrm{PL}}\right), & \text { for } F_{\mathrm{PL}} \leq F_{\mathrm{brk}, \mathrm{hi}}, \\ X\left(F_{\mathrm{brk}, \mathrm{hi}}\right), & \text { for } F_{\mathrm{PL}}>F_{\mathrm{brk}, \mathrm{hi}}\end{cases}$

Interestingly, the best-fit break fluxes for $\Gamma\left(F_{\mathrm{PL}}\right)$ and $F_{\mathrm{BB}}\left(F_{\mathrm{PL}}\right)$ are the same within their uncertainties. Thus, we tied the break fluxes, $F_{\text {brk,hi }}$, for both parameter evolutions together. The observed parameter correlations can be described even better if we allow for an additional break of the photon index, $\Gamma$, to a constant at lower fluxes, $F_{\text {brk,lo }}$, which we had noticed already in K14,

low-brkn: $X^{\prime}\left(F_{\mathrm{PL}}\right)= \begin{cases}X\left(F_{\mathrm{brk}, \mathrm{lo}}\right), & \text { for } F_{\mathrm{PL}}<F_{\mathrm{brk}, \mathrm{lo}}, \\ X\left(F_{\mathrm{PL}}\right), & \text { for } F_{\mathrm{PL}} \geq F_{\mathrm{brk}, \mathrm{lo}}\end{cases}$

This model for the spectral evolution of GRO J1008-57 including two breaks at different luminosity levels provides a good description of the data. The corresponding fit parameters are listed in Table 3.

Since we interpret the artificial breaks in the model function as transitions between different accretion regimes, the statistical significance of their detection is crucial for our conclusion. The least prejudiced way to derive the significance of these model components is a Monte Carlo approach. Therefore, we simulated $1.77 \times 10^{6}$ data sets for both $\Gamma\left(F_{\mathrm{PL}}\right)$ and $F_{\mathrm{BB}}\left(F_{\mathrm{PL}}\right)$ based on our best-fit model without any breaks. A Gaussian randomization of the individual data points with their respective uncertainty has been applied for each simulated data set. The resulting data sets were then fitted both with the model with and without the two breaks. If the $\chi^{2}$ difference of those two fits was larger than the one obtained from fitting our measured data, we recorded this as a false-positive detection. The fraction of false-positive detections in the complete simulation directly translates to the significance of the modeled break. We find a significance of the high-luminosity break in $F_{\mathrm{BB}}$ of $\geq 5 \sigma$ as a lower limit. The highand low-luminosity breaks of the photon index, $\Gamma$, are significant at the $2.35 \sigma$ and $3.73 \sigma$ level, respectively. We thus conclude that the high-luminosity break in $F_{\mathrm{BB}}$ and the low-luminosity break in $\Gamma$ are most likely real, while the high-luminosity break of $\Gamma$ is only moderately significant. We note that the chosen additional uncertainties as described above move these significances to the conservative side. For instance, the significane of the lowluminosity break in $\Gamma$ increases to 4.12 once no systematic uncertainty is added and only RXTE data are taken into account (this is the only data set relevant at this luminosity level, see Fig. 6).

We stress that other models for the spectral evolution of GRO J1008-57 as a function of luminosity might provide an equivalent description of the data. However, regardless of the chosen model or approach (e.g., investigating hardness ratios) we significantly detect changes near $F_{\text {brk,lo }}$ and $F_{\text {brk,hi }}$. We do not claim that our (phenomenological) model or the way in handling systematic uncertainties is generally valid. Thus, the spectral shape of GRO J1008-57 for any flux as predicted by our model should be taken with care.

Accretion regimes in GRO J1008-57. The detailed analysis of the evolution of the photon index and black body flux presented in Sect. 5 revealed two flux levels, where a change in the evolution is happening (see Fig. 6). At fluxes below $F_{\text {brk,lo }} \sim$ $10^{-10} \mathrm{erg} \mathrm{s}^{-1} \mathrm{~cm}^{-2}$ (between 15-50 keV) the photon index seems to be independent of flux and stays constant. For higher fluxes, the photon index starts to harden, while the black body flux increases. Once the flux exceeds $F_{\text {brk,hi }} \sim 3 \times 10^{-9} \mathrm{erg} \mathrm{s}^{-1} \mathrm{~cm}^{-2}$ a saturation effect is observed, where the photon index is no longer hardening and the black body does not increase in flux further. In addition, during the giant 2012 November outburst (epoch 1a and 1b), when GRO J1008-57 reached its highest known flux so $\mathrm{far}^{3}$, another soft component below $3 \mathrm{keV}$ shows up in the Suzaku spectrum (the second black body, see Sect. 3.1). This feature is not detected in any other observation at lower fluxes. We interpret these facts as observational evidence for three different accretion regimes in GRO J1008-57.

In order to connect these different accretion regimes of GRO J1008-57 with the physics of the accretion process, we have compared our findings with recent theoretical investigations by Becker et al. (2012), Postnov et al. (2015a), and Mushtukov et al. (2015a). These authors estimate the so-called critical luminosity, which can be interpreted as a transition between two accretion regimes: above this luminosity the radiation pressure inside the column dominates the deceleration of the infalling plasma, while Coloumb interactions dominate at lower luminosities. In addition, Becker et al. (2012) expect

3 The physical flux of GRO J1008-57 within 20 and $50 \mathrm{keV}$ during its discovery outburst in 1993 was $2.2 \times 10^{-9} \mathrm{erg} \mathrm{s}^{-1} \mathrm{~cm}^{-2}$ (Shrader et al. 1999). 


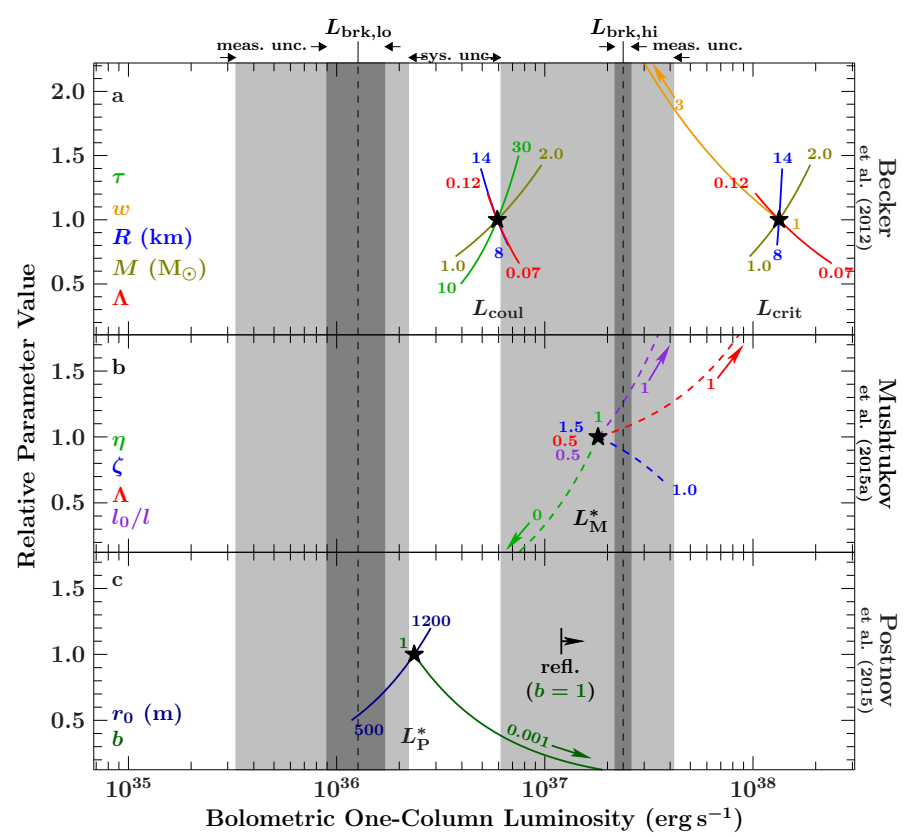

Fig. 7. Comparison of the derived luminosities at the breaks of the spectral evolution ( $L_{\text {brk,lo }}$ and $L_{\text {brk,hi }}$, vertical dashed lines) with $L_{\text {coul }}$ and $L_{\text {crit }}$ of Becker et al. (2012, Eqs. (A.1) and (A.2); panel a), $L_{\mathrm{M}}^{*}$ of Mushtukov et al. (2015a, Eq. (A.3); b), and $L_{\mathrm{P}}^{*}$ of Postnov et al. (2015a, Eq. (A.4); c). The dark gray bands correspond to the $90 \%$ statistical uncertainties of the measured break fluxes (meas. unc). The larger widths in light gray are the uncertainties when deriving luminosities from the observed fluxes as described in the text (sys. unc.). The black stars are the theoretically expected values for typical neutron star parameters and $E_{\mathrm{cyc}}$ as found by NUSTAR and corrected for the gravitational redshift. The colored lines represent changes in the neutron star parameters with their relative value along the $y$-axis. The minimum and maximum absolute values are marked at the line endings. The black arrow in panel (c) marks the luminosity above which Postnov et al. (2015a) expect reflected radiation from the neutron star's surface to contribute significantly. The dependencies of the parameters in panel (b) (dashed lines) are assumed to be linear (see text for details).

these Coloumb interactions to be unable to stop the material to rest for even lower luminosities. This so-called Coloumb braking luminosity would mark another transition between accretion regimes. The theoretical assumptions and calculations of these three works result, however, in differences by orders of magnitude. More details and the actual equations for the transition luminosities are given in Appendix A.

Figure 7 compares the luminosities of GRO J1008-57 at the observed breaks in the spectral parameter evolution, $L_{\text {brk,lo }}$ and $L_{\text {brk,hi }}$, with the theoretically expected luminosities at the transition between different accretion regimes after Becker et al. (2012), Postnov et al. (2015a), and Mushtukov et al. (2015a) (Eqs. (A.1)-(A.3)). To derive the bolometric luminosities in the neutron star's rest-frame, $L$, from the observed fluxes, $F_{\text {brk,lo }}$ and $F_{\text {brk,hi }}$, we need to

a) extrapolate the model to the full electromagnetic spectrum in order to calculate the unabsorbed bolometric flux, $F^{\star}$;

b) correct for the gravitational redshift, $z$;

c) take into account a factor, $g$, describing the emission geometry;

d) and, correct for the distance, $d$, to the source.

In summary,

$L=g d^{2}(1+z)^{2} F^{\star}\left(F_{\mathrm{PL}}\right)$.
We describe the details of the corrections a) to d) in the following and discuss their uncertainties.

a) The unabsorbed bolometric flux, $F^{\star}$, is a non-linear function of the power-law flux, $F_{\mathrm{PL}}$. We have used our spectral evolution model (see Table 3 ) in order to extrapolate the observed spectral shape to the 0.01 to $100 \mathrm{keV}$ energy range. From this extended spectrum we determine the bolometric flux. The uncertainty of the extrapolation is caused by the unknown spectral shape outside of the observed energy band. Here, we assume a thermal Comptonization spectrum ${ }^{4}$ below $1 \mathrm{keV}$, that is, below the Swift-XRT and Suzaku-XIS sensitivities. Furthermore, the uncertainty in the estimated bolometric flux takes the uncertainty of the measured absorption column density, $N_{\mathrm{H}}$, into account. It is unlikely that the observed $\mathrm{X}$-ray absorption mimics an actual Comptonization turn-over since we do not observe a change in $N_{\mathrm{H}}$ with time or flux in GRO J1008-57(K13). Due to the exponential rollover at higher energies the spectral shape above $\sim 80 \mathrm{keV}$ (NuSTAR and Suzaku GSO) does not influence the bolometric flux significantly. We found that the extrapolation of the energy band results in a $0.01-100 \mathrm{keV}$ flux uncertainty of $15 \%$ when comparing thermal Comptonization with the power-law spectrum used for spectral analysis (see Sect. 3).

b) The gravitational redshift, $1+z=\left(1-2 G M / R c^{2}\right)^{-1 / 2}$ with the mass, $M$, and radius, $R$, of the neutron star results in an observed luminosity of $L_{\mathrm{obs}}=L /(1+z)^{2}$ compared to the intrinsic one (Thorne 1977). For a typical neutron star with $M=1.4 M_{\odot}$ and $R=12 \mathrm{~km}$, we find $z \approx 0.235$. Assuming $\Delta M=0.5 M_{\odot}$ and $\Delta R=6 \mathrm{~km}$ we find an uncertainty of $\Delta z=0.1$ in $z$, which corresponds to a $17 \%$ uncertainty in the derived intrinsic luminosity.

c) The emission geometry of GRO J1008-57 is unknown and, thus, we assume isotropic emission of each pole into its hemisphere, that is, $g=2 \pi$ (the theoretical expectations apply to one pole only). According to Martínez-Núñez et al. (2017), who estimate the effects of light bending (e.g., Kraus 2001), the real luminosity for a typical fan beam accretion geometry can differ by $\sim 25 \%$ relative to the derived luminosity assuming isotropic emission. Due to the higher accretion rate in BeXRBs compared to low-mass X-ray binaries (LMXBs), which usually emit via a pencil beam geometry (see, e.g., Basko \& Sunyaev 1975), the fan beam is a justified geometry for GRO J1008-57. Thus, we assume a systematic uncertainty of $25 \%$ due to the unknown emission geometry.

d) The distance to the source was determined as $d=5.8(5) \mathrm{kpc}$ by Riquelme et al. (2012), which propagates to a further $17 \%$ uncertainty in the luminosity.

Using Eq. (6) we converted the measured fluxes where we observed changes in the spectral behavior of GRO J1008-57, $F_{\text {brk,lo }}$ and $F_{\text {brk,hi }}$, into the luminosities shown in Fig. $7, L_{\text {brk,lo }}$ and $L_{\text {brk,hi }}$ (vertical dashed lines). Besides the corresponding measurement uncertainties of these luminosities (dark gray bands) we added a systematic uncertainty of $74 \%$ to the data (light gray bands) which corresponds to the sum of the additional sources of uncertainty, a-d, as described above ${ }^{5}$. In order

4 compTT (Titarchuk 1994): seed photons of $k T=1.883 \mathrm{keV}$, plasma temperature of $E_{\text {fold }}=15.9 \mathrm{keV}$, plasma optical depth of 20 (Becker et al. 2012), and normalized to match the source's spectrum at $1 \mathrm{keV}$.

5 The uncertainties of the contributions a-d are mainly due to systematics caused by, e.g., extrapolation or theoretical assumptions. Thus, we chose to add their corresponding uncertainties directly instead of adding them in quadrature. 
to calculate the theoretical luminosities after Eqs. (A.1)-(A.3) (black stars), we assumed typical neutron star parameters (see Eqs. (A.1)-(A.3) and the respective references) with the exception of the surface cyclotron line energy, $E_{\star}$, which we assumed to be the redshift corrected cyclotron line energy, $E_{\star}=$ $(1+z) E_{\text {cyc }}=90 \mathrm{keV}$, with the mean value of $E_{\text {cyc }}$ as listed in Table 2. The dependencies of the different luminosities on the neutron star parameters are shown by the colored lines. Because Mushtukov et al. (2015a) solved Eq. (A.3) numerically they presented the dependencies of their critical luminosity, $L_{\mathrm{M}}^{*}$, on the neutron star parameters in their Fig. 5 instead of providing an analytical equation. From this figure, we have extracted the values of the critical luminosity assuming the same redshift corrected cyclotron line energy as above. In order to include the dependency of the critical luminosity, $L_{\mathrm{M}}^{*}$ (Eq. (A.3)), on neutron star parameters in Fig. 7, we assumed a linear dependency between the extracted values. By drawing the resulting dependencies as dashed lines (panel b) we stress that this is for illustrating the basic dependence, that is, its sign, only.

The facts from Fig. 6, which shows the spectral parameter evolution of GRO J1008-57, and Fig. 7, which compares the changes in this parameter evolution at $L_{\text {brk,lo }}$ and $L_{\text {brk,hi }}$ with recent theoretical investigations, can be summarized as follows.

- Between the observed luminosities, $L_{\text {brk,lo }}$ and $L_{\text {brk,hi }}$, the spectrum of GRO J1008-57 is hardening with increasing luminosity. This is in line with theoretical expectations by Postnov et al. (2015a) for sources in the subcritical accretion regime.

- If $L_{\text {brk,hi }}$ can indeed be associated with the source exceeding the critical luminosity, then parameter combinations can be found such that each of the three theories summarized in Appendix A predict the expected value for this luminosity correctly:

- $L_{\text {brk,hi }} \sim L_{\text {crit }}$ for $w>1.9$ after Becker et al. (2012, Eq. (A.2)), that is, the spectrum inside the column is a mixture between a Plank spectrum $(w=3)$ and pure Comptonized bremsstrahlung radiation $(w=1)$.

- $L_{\text {brk,hi }} \sim L_{\mathrm{M}}^{*}$ after Mushtukov et al. (2015a, Eq. (A.3)) for canonical neutron star parameters.

- $L_{\text {brk,hi }} \sim L_{\mathrm{P}}^{*}$ after Postnov et al. (2015a, Eq. (A.4)) for $b \ll 1$, that is, a hollow accretion column. We note that Eq. (A.4) depends equally on $b$ and $\kappa_{\perp} / \kappa_{\mathrm{T}}$, that is, the same effect is achieved for $\kappa_{\perp} / \kappa_{\mathrm{T}} \ll 1$.

- The change of the spectral evolution at $L_{\text {brk,lo }}$ is consistent with $L_{\mathrm{P}}^{*}$ after Postnov et al. (2015a, Eq. (A.4)) for a filled accretion column with a radius of $r_{0} \leq 935 \mathrm{~m}$. The corresponding luminosity around $10^{36} \mathrm{erg} \mathrm{s}^{-1}$ is, however, too low for the transition to supercritical accretion as expected by all these theories. Even Postnov et al. (2015a) expect this transition to occur around $10^{37} \mathrm{erg} \mathrm{s}^{-1}$.

- The ratio between the Coulomb braking and the critical luminosity, $L_{\text {coul }} / L_{\text {crit }}$, after Becker et al. (2012, Eqs. (A.1) and (A.2)) matches almost perfectly the observed ratio $L_{\text {brk,lo }} / L_{\text {brk,hi. The individual theoretical luminosities for }}$ canonical neutron star parameters are, however, a factor of $\sim 6$ higher than compared to observed ones.

- The observed spectral change at high luminosities, $L_{\mathrm{brk}, \mathrm{hi}}$, is also consistent with the saturation of the hardness as expected by Postnov et al. (2015a) due to reflection from the neutron star's surface in case of a filled accretion column.

We have further investigated the dilemma that $L_{\text {brk,hi }}$ is consistent with reflection from a filled accretion column, but also with $L_{\mathrm{P}}^{*}$ for a hollow accretion column after Postnov et al. (2015b).
From our best-fit parameter evolution (see Table 3) we calculated the hardness ratio using the same energy bands as Postnov et al. (2015b, 5-12 keV over $1.3-3 \mathrm{keV}$ ). The resulting evolution of the hardness ratio does not increase above a hardness of $\sim 6$. This is in very good agreement with the expected value for the hardness ratio in case of a filled accretion column, whereas a hardness in the range of 10-16 is expected for a hollow accretion column (see Figs. 6 and 7 of Postnov et al. 2015b). Furthermore, the derived hardness saturates above a (one-column) accretion rate of $1 \times 10^{17} \mathrm{~g} \mathrm{~s}^{-1}$, while the evolution of the hardness shown in Fig. 6 of Postnov et al. (2015b) suggests a saturation above $5-7 \times 10^{17} \mathrm{~g} \mathrm{~s}^{-1}$. This value scales with the height of the filled accretion column, which itself anti-correlates with the magnetic field strength at a given mass accretion rate (K. Postnov, priv. comm.). The required magnetic field strength to achieve a match between the observed $L_{\text {brk,hi }}$ and the expected saturation of the hardness due to reflection is, however, far lower than compared to GRO J1008-57's CRSF. In summary, the match of $L_{\mathrm{P}}^{*}$ with $L_{\text {brk,hi }}$ in the case of a hollow column is ruled out by the observed hardness ratio. Once a filled column is assumed, the overall hardness ratio matches the predicted value. The dependence of $L_{\mathrm{P}}^{*}$ on the luminosity due to reflection at the neutron star's surface requires, however, a much weaker magnetic field than what is found for GRO J1008-57.

We notice that the observed break at high luminosities, $L_{\text {brk,hi }}$, agrees well with the critical luminosity, $L_{\mathrm{M}}^{*}$, as predicted by Mushtukov et al. (2015a), at least for the case of GRO J1008-57. The theories by Postnov et al. (2015b) and Becker et al. (2012) have difficulties explaining our observations since unlikely parameter combinations are necessary in order to achieve a match with their predicted critical luminosities, $L_{\mathrm{P}}^{*}$ and $L_{\text {crit }}$, respectively (see Fig. 7). The large systematic uncertainties when deriving luminosities from observational data as discussed above do not, however, allow us to favor unequivocally one of the discussed theories for the prediction of the critical luminosity. Especially, the observed change of the spectral evolution of GRO J1008-57 at low luminosities, $L_{\text {brk,lo }}$, cannot be explained by either theory. Furthermore, drawing general conclusions about these theories is statistically questionable as only observational data of a single source are used. In future work, a detailed study of the recently claimed accretion regimes in 4 U 1901+03 (Reig \& Milonaki 2016) and V 0332+53 (Doroshenko et al. 2016) combined with the results by Reig \& Nespoli (2013) for various sources could help clarify this question. It should be noted, however, that thorough analysis methods are required and systematic effects (caused by detector calibration and due to the conversion to luminosities) have to be taken into account as, for example, discussed here. Nevertheless, the existence of different accretion regimes, which are driven by the mass accretion rate, seems to be confirmed.

In summary, however, the theories allow us to associate the following physical accretion regimes with the observed changes in the spectral evolution of GRO J1008-57:

- $L>L_{\text {brk,hi }} \sim 2 \times 10^{37} \mathrm{erg} \mathrm{s}^{-1}$ : supercritical accretion, where radiation dominates the deceleration of the infalling plasma and stops the hardening of the X-ray spectrum.

- $10^{36} \mathrm{erg} \mathrm{s}^{-1} \sim L_{\text {brk,lo }}<L<L_{\text {brk,hi }}$ : subcritical accretion regime, where Comptonization effects scale with the mass accretion rate.

- $L<L_{\text {brk,lo: }}$ very low subcritical regime, where physical effects depend only marginally on the mass accretion rate.

Further investigations of the source are required to confirm or reject these conclusions. In order to proceed, however, working 
self-consistent models should be applied to the spectra of GRO J1008-57 to reveal the evolution of its physical parameters with luminosity. Additionally, high-SNR observations of the source at very low $\left(L<L_{\text {brk,lo }}\right)$ and extremely high luminosities $\left(L>L_{\mathrm{brk}, \mathrm{hi}}\right)$ are required to investigate the existence of the different accretion regimes as proposed here. Finally, theory predicts different flux dependencies of the CRSF parameters depending on the accretion regime, which could be probed for GRO J1008-57 with future hard X-ray missions with a sufficiently high effective area around $100 \mathrm{keV}$.

Acknowledgements. M.K. acknowledges support by the Bundesministerium für Wirtschaft und Technologie under Deutsches Zentrum für Luft- und Raumfahrt grants 50OR1113 and 50OR1207. We appreciate the useful discussions with K. Postnov and M. Gornostaev about accretion column physics. All figures shown in this paper were produced using the SLXfig module, developed by John E. Davis. We thank the Suzaku and NuSTAR teams for accepting our proposals and performing the observations. Finally, we thank the referee for her/his valuable comments, which helped improving the quality of our paper.

\section{References}

Basko, M. M., \& Sunyaev, R. A. 1975, A\&A, 42, 311

Basko, M. M., \& Sunyaev, R. A. 1976, MNRAS, 175, 395

Becker, P. A., \& Wolff, M. T. 2007, ApJ, 654, 435

Becker, P. A., Klochkov, D., Schönherr, G., et al. 2012, A\&A, 544, A123

Bellm, E. C., Fürst, F., Pottschmidt, K., et al. 2014, ApJ, 792, 108

Boldt, E. 1987, in Observational Cosmology, eds. A. Hewitt, G. Burbidge, \&

L. Z. Fang (Cambridge: Cambridge Univ. Press), IAU Symp., 124, 611

Bradt, H. V., Rothschild, R. E., \& Swank, J. H. 1993, A\&AS, 97, 355

Burrows, D. N., Hill, J. E., Nousek, J. A., et al. 2005, Space Sci. Rev., 120, 165

Caballero, I., \& Wilms, J. 2012, Mem. Soc. Astron. It., 83, 230

Caballero, I., Santangelo, A., Kretschmar, P., et al. 2008, A\&A, 480, L17

Caballero, I., Pottschmidt, K., Marcu, D. M., et al. 2013, ApJ, 764, L23

Capalbi, M., Perri, M., Saija, B., et al. 2005, NASA Goddard Space Flight Center, The SWIFT XRT Data Reduction Guide, Version 1.2, http://swift.gsfc. nasa.gov/analysis/

Coe, M. J., Roche, P., Everall, C., et al. 1994, MNRAS, 270, L57

Coe, M. J., Bird, A. J., Hill, A. B., et al. 2007, MNRAS, 378, 1427

Doğan, S., Nixon, C., King, A., \& Price, D. J. 2015, MNRAS, 449, 1251

Doroshenko, V., Tsygankov, S. S., Mushtukov, A. A., et al. 2016, MNRAS, 466 2143

Farinelli, R., Ceccobello, C., Romano, P., \& Titarchuk, L. 2012, A\&A, 538, A67

Farinelli, R., Ferrigno, C., Bozzo, E., \& Becker, P. A. 2016, A\&A, 591, A29

Ferrigno, C., Falanga, M., Bozzo, E., et al. 2011, A\&A, 532, A76

Finger, M. H., Camero-Arranz, A., Kretschmar, P., Wilson, C., \& Patel, S. 2006, in BAAS, 38, 359

Fürst, F., Pottschmidt, K., Wilms, J., et al. 2014, ApJ, 780, 133

Gehrels, N., Chincarini, G., Giommi, P., et al. 2004, ApJ, 611, 1005

Gehrels, N., Chincarini, G., Giommi, P., et al. 2005, ApJ, 621, 558

Godet, O., Beardmore, A. P., Abbey, A. F., et al. 2007, in UV, X-ray, and Gamma-ray Space Instrumentation for Astronomy XV, eds. O. H. W. Siegmund, \& M. A. Gummin, SPIE Conf. Ser., 6686, 66860

Houck, J. C., \& Denicola, L. A. 2000, in Astronomical Data Analysis Software and Systems IX, eds. N. Manset, C. Veillet, \& D. Crabtree, ASP Conf. Ser., 216,591

Hurkett, C. P., Vaughan, S., Osborne, J. P., et al. 2008, ApJ, 679, 587

ISAS/JAXA, et al. 2013, The Suzaku Data Reduction Guide, Version 5, http: //heasarc.gsfc.nasa.gov/docs/suzaku/analysis/abc/

Jahoda, K., Swank, J. H., Giles, A. B., et al. 1996, in UV, X-ray, and Gamma-ray Space Instrumentation for Astronomy VII, eds. O. H. W. Siegmund, \& M. A. Gummin, SPIE Conf. Ser., 2808, 59
Kirsch, M. G., Briel, U. G., Burrows, D., et al. 2005, in UV, X-Ray, and GammaRay Space Instrumentation for Astronomy XIV, ed. O. H. W. Siegmund, SPIE Conf. Ser., 5898, 22

Kokubun, M., Fukazawa, Y., Idesawa, E., et al. 1999, IEEE Trans. Nucl. Sci., 46, 371

Kokubun, M., Makishima, K., Takahashi, T., et al. 2007, PASJ, 59, 53

Koyama, K., Tsunemi, H., Dotani, T., et al. 2007, PASJ, 59, 23

Kraus, U. 2001, ApJ, 563, 289

Kretschmar, P., Salazar, E., Laplace, E., et al. 2015, ATel, 6917, 1

Krimm, H. A., Holland, S. T., Corbet, R. H. D., et al. 2013, ApJS, 209, 14

Kühnel, M., Müller, S., Kreykenbohm, I., et al. 2013, A\&A, 555, A95

Kühnel, M., Müller, S., Kreykenbohm, I., et al. 2014, in Physics at the Magnetospheric Boundary, EPJ Web Conf., 64, 06003

Kühnel, M., Müller, S., Kreykenbohm, I., et al. 2015, Acta Polytechnica, 55, 123

Kühnel, M., Falkner, S., Grossberger, C., et al. 2016, Acta Polytechnica, 56, 41

Lamb, F. K., Pethick, C. J., \& Pines, D. 1973, ApJ, 184, 271

Leahy, D. A., Darbro, W., Elsner, R. F., et al. 1983, ApJ, 266, 160

Madsen, K. K., Beardmore, A. P., Forster, K., et al. 2017, AJ, 153, 2

Makishima, K., Mihara, T., Ishida, M., et al. 1990, ApJ, 365, L59

Marcu, D. M., Pottschmidt, K., Gottlieb, A. M., et al. 2015, in Proc. the 10th INTEGRAL Workshop "A Synergistic View of the High Energy Sky" (INTEGRAL 2014), PoS

Marcu-Cheatham, D. M., Pottschmidt, K., Kühnel, M., et al. 2015, ApJ, 815, 44

Martínez-Núñez, S., Kretschmar, P., Bozzo, E., et al. 2017, Space Sci. Rev., in press DOI: 10.1107/s11214-017-0340-1

Mitsuda, K., Bautz, M., Inoue, H., et al. 2007, PASJ, 59, 1

Mushtukov, A. A., Suleimanov, V. F., Tsygankov, S. S., \& Poutanen, J. 2015a, MNRAS, 447, 1847

Mushtukov, A. A., Tsygankov, S. S., Serber, A. V., Suleimanov, V. F., \& Poutanen, J. 2015b, MNRAS, 454, 2714

Nakajima, M., Mihara, T., Sugizaki, M., et al. 2012, ATel, 4420, 1

Nakajima, M., Ohtsuki, H., Ueno, S., et al. 2014a, ATel, 6465, 1

Nakajima, M., Sugizaki, M., Mihara, T., et al. 2014b, ATel, 6819, 1

Nowak, M. A., Hanke, M., Trowbridge, S. N., et al. 2011, ApJ, 728, 13

Okazaki, A. T., \& Negueruela, I. 2001, A\&A, 377, 161

Okazaki, A. T., Hayasaki, K., \& Moritani, Y. 2013, PASJ, 65, 41

Perri, M., Puccetti, S., Spagnuol, N., et al. 2015, The NuSTAR Data Analysis Software Guide, Version 1.8.0, http://heasarc.gsfc.nasa.gov/docs/ nustar/analysis/nustar_swguide.pdf

Postnov, K., Staubert, R., Santangelo, A., et al. 2008, A\&A, 480, L21

Postnov, K. A., Gornostaev, M. I., Klochkov, D., et al. 2015a, MNRAS, 452, 1601

Postnov, K. A., Mironov, A. I., Lutovinov, A. A., et al. 2015b, MNRAS, 446, 1013

Priedhorsky, W. C., \& Terrell, J. 1983, ApJ, 273, 709

Reig, P., \& Milonaki, F. 2016, A\&A, 594, A45

Reig, P., \& Nespoli, E. 2013, A\&A, 551, A1

Riquelme, M. S., Torrejón, J. M., \& Negueruela, I. 2012, A\&A, 539, A114

Schönherr, G., Schwarm, F.-W., Falkner, S., et al. 2014, A\&A, 564, L8

Serlemitsos, P. J., Soong, Y., Chan, K.-W., et al. 2007, PASJ, 59, 9

Shrader, C. R., Sutaria, F. K., Singh, K. P., \& Macomb, D. J. 1999, ApJ, 512, 920

Staubert, R., Shakura, N. I., Postnov, K., et al. 2007, A\&A, 465, L25

Stollberg, M. T., Finger, M. H., Wilson, R. B., et al. 1993, IAU Circ., 5836, 1

Takahashi, T., Abe, K., Endo, M., et al. 2007, PASJ, 59, 35

Thorne, K. S. 1977, ApJ, 212, 825

Titarchuk, L. 1994, ApJ, 434, 570

Tomsick, J. A., Nowak, M. A., Parker, M., et al. 2014, ApJ, 780, 78

Tsujimoto, M., Guainazzi, M., Plucinsky, P. P., et al. 2011, A\&A, 525, A25

Verner, D. A., Ferland, G. J., Korista, K. T., \& Yakovlev, D. G. 1996, ApJ, 465, 487

Walton, D. J., Tomsick, J. A., Madsen, K. K., et al. 2016, ApJ, 826, 87

Wilms, J., Allen, A., \& McCray, R. 2000, ApJ, 542, 914

Wilson, R. B., Harmon, B. A., Fishman, G. J., et al. 1994, in The Evolution of

X-ray Binaries, eds. S. Holt, \& C. S. Day, AIP Conf. Ser., 308, 451

Wolff, M. T., Becker, P. A., Gottlieb, A. M., et al. 2016, ApJ, 831, 194

Yamamoto, T., Mihara, T., Sugizaki, M., et al. 2013, ATel, 4759, 1

Yamamoto, T., Mihara, T., Sugizaki, M., et al. 2014, PASJ, 66, 59 


\section{Appendix A: Critical luminosity}

At the critical luminosity of an accreting neutron star the radiation pressure generated at the base of the accretion column contributes significantly to the deceleration of the infalling plasma (Basko \& Sunyaev 1975). In the past many attempts have been made to derive this critical luminosity theoretically. Three of these theories are summarized in the following.

The X-ray spectra of several accreting neutron stars exhibit so-called cyclotron resonant scattering features (CRSFs; see, e.g., Caballero \& Wilms 2012, for a review). These absorption features arise from transitions between the Landau levels of electrons in the accreted plasma, which are quantized due to the strong magnetic field of these neutron stars on the order of $10^{12} \mathrm{G}$. The observed cyclotron line energy, $E_{\mathrm{cyc}}$, is found to show a positive or negative correlation with the mass accretion rate, $\dot{M}$ (see, e.g., Caballero \& Wilms 2012; and Becker et al. 2012, and references therein).

In order to explain theoretically the different types of behavior of CRSF energy with luminosity, Becker et al. (2012) investigated the characteristic height of the X-ray emission region in the accretion column as a function of the mass accretion rate. These authors proposed different $\dot{M}$ regimes in which the height of the shock positively correlates (negative $E_{\text {cyc }}$ correlation) or negatively correlates (positive $E_{\text {cyc }}$ correlation) with $\dot{M}$. The luminosities at which the transitions between these regimes occur, are known as $L_{\text {coul }}$ and $L_{\text {crit }}$, and are given by (Eqs. (59) and (55) of Becker et al. 2012)

$$
\begin{aligned}
L_{\text {coul }}= & 1.23 \times 10^{37} \mathrm{erg} \mathrm{s}^{-1}\left(\frac{\Lambda}{0.1}\right)^{-7 / 12}\left(\frac{\tau_{\star}}{20}\right)^{7 / 12} \\
& \times\left(\frac{M_{\star}}{1.4 M_{\odot}}\right)^{11 / 8}\left(\frac{R_{\star}}{10 \mathrm{~km}}\right)^{-13 / 24}\left(\frac{E_{\star}}{10 \mathrm{keV}}\right)^{-1 / 3}
\end{aligned}
$$

and

$$
\begin{aligned}
L_{\text {crit }}= & 1.28 \times 10^{37} \operatorname{erg~s}^{-1}\left(\frac{\Lambda}{0.1}\right)^{-7 / 5} w^{-28 / 15}\left(\frac{M_{\star}}{1.4 M_{\odot}}\right)^{29 / 30} \\
& \times\left(\frac{R_{\star}}{10 \mathrm{~km}}\right)^{1 / 10}\left(\frac{E_{\star}}{10 \mathrm{keV}}\right)^{16 / 15} \cdot
\end{aligned}
$$

Here, $\Lambda$ is a parameter describing the accretion geometry $(\Lambda=1$ for spherical accretion from a wind and $\Lambda<1$ for accretion from a disk, see also Lamb et al. 1973), $\tau_{\star}$ is the Thomson optical depth, $M_{\star}$ and $R_{\star}$ are the mass and radius of the neutron star, respectively, $E_{\star}$ is the CRSF energy related to the surface magnetic field, and $w$ is a parameter describing the spectral shape inside the column ( $w=1$ for a Bremsstrahlung spectrum and $w=3$ for a Planck spectrum). In the model of Becker et al. (2012), the in-falling matter is decelerated by passing through a radiation dominated shock. Above the so-called critical luminosity, $L_{\text {crit }}$ (in the supercritical accretion regime), the radiation pressure alone is able to stop the matter above the neutron star's surface. Below this luminosity (in the subcritical regime), the radiationdominated shock still exists, but the final deceleration occurs via Coulomb braking within the accretion flow. At very low luminosities, below a characteristic luminosity, $L_{\text {coul }}$, Coulomb interactions are no longer sufficient to stop the matter. The detailed mechanism to decelerate the matter to rest is not yet clear (see., e.g. Fürst et al. 2014).
Alternatively, Mushtukov et al. (2015a) calculate the critical luminosity, that is, where the supercritical accretion sets in taking resonant scattering and photon polarization into account for the first time. For a circular hotspot on the neutron star's surface, this luminosity is given by (Basko \& Sunyaev 1975, 1976; Eq. (7) in Mushtukov et al. 2015a)

$$
L_{\mathrm{M}}^{*} \approx 3.7 \times 10^{36}\left(\frac{\kappa_{\mathrm{T}}}{\kappa_{\mathrm{eff}}}\right)\left(\frac{d}{10^{5} \mathrm{~cm}}\right)\left(\frac{R_{\star}}{10^{6} \mathrm{~cm}}\right)^{-1}\left(\frac{M_{\star}}{1 M_{\odot}}\right) \mathrm{erg} \mathrm{s}^{-1},
$$

where $\kappa_{\mathrm{eff}}$ is the effective scattering cross-section and $d$ is the diameter of the hot spot. The key issue here is the calculation of the effective scattering cross-section, $\kappa_{\text {eff }}$, which is solved by Mushtukov et al. (2015a) numerically assuming specific accretion column geometries for wind- and disk-accretion, a linear velocity profile, and black body seed photons. Although these authors assumed a radiation dominated shock to exist for luminosities above the critical luminosity, $L_{\mathrm{M}}^{*}$, they argue that the value of $L_{\mathrm{M}}^{*}$ is mainly determined by the effective cross-section, $\kappa_{\mathrm{eff}}$, due to resonant scattering of electrons. In contrast to Becker et al. (2012), they expect the X-ray emission region to settle down on the neutron star's surface for luminosities below the critical luminosity, $L_{\mathrm{M}}^{*}$. In this subcritical regime, Mushtukov et al. (2015b) do not expect a radiation dominated shock to be formed. Instead, they explain the positive correlation of $E_{\text {cyc }}$ with $\dot{M}$ by the (still) relativistic velocity of the in-falling plasma near the surface, which results in a Doppler shift of the CRSF energy.

Another recent theoretical investigation by Postnov et al. (2015a) calculates the X-ray spectrum in the supercritical regime using the radiation diffusion approximation and assuming the emission emerging from the walls of the accretion column (known as the fan beam geometry). In this scenario the accretion column is assumed to be optically thick and the infalling matter is decelerated by a radiative shock similar to Becker et al. (2012). The minimum luminosity, $L_{\mathrm{P}}^{*}$, at which an optically thick accretion column appears, is given by (Eq. (5) of Postnov et al. 2015a)

$L_{\mathrm{P}}^{*} \approx 2.36 \times 10^{36} \mathrm{erg} \mathrm{s}^{-1}\left(\frac{r_{0}}{10^{5} \mathrm{~cm}}\right)\left(\frac{b \kappa_{\perp}}{\kappa_{T}}\right)^{-1}$,

with the radius $r_{0}$ of the accretion column, the cross-sections $\kappa_{\perp}$ and $\kappa_{T}$, and the thickness of the column walls, $0<b \leq 1$, relative to its radius ( $b=1$ corresponds to a filled accretion column, while $b \ll 1$ is a hollow column). In principle, their derived luminosity, $L_{\mathrm{P}}^{*}$, describes the same physical condition as $L_{\text {crit }}$ after Becker et al. (2012) and $L_{\mathrm{M}}^{*}$ after Mushtukov et al. (2015a). For luminosities $L<L_{\mathrm{P}}^{*}$, that is, in the subcritical regime, Postnov et al. (2015a) showed that the observed X-ray spectrum hardens with increasing mass accretion rate, $\dot{M}$, due to an increase in the Comptonization parameter. Finally, at very high luminosities around (3-7) $\times 10^{37} \mathrm{erg} \mathrm{s}^{-1}$ they observe a saturation in the hardness in some accreting pulsars using data from the All Sky Monitor (ASM) onboard the Rossi X-ray Timing Explorer (RXTE Bradt et al. 1993). They are able to explain this behavior by including reflected radiation from the neutron star's surface, which is illuminated by the Doppler-boosted radiation of the column walls, while the spectrum continues to harden for increasing luminosities. Thus, in the model of Postnov et al. (2015a), the observed saturation is a purely geometric effect. 